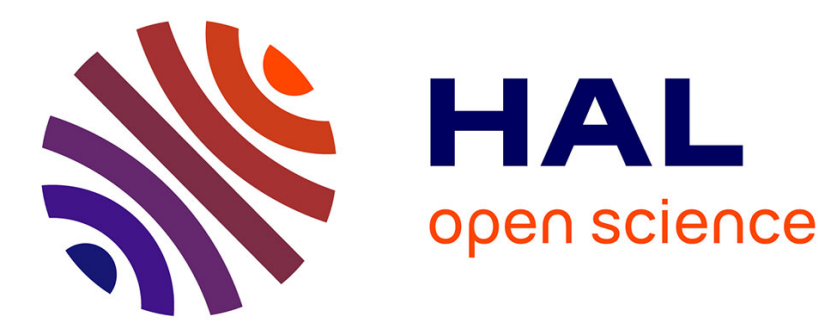

\title{
Recent Advanced Statistical Background Modeling for Foreground Detection - A Systematic Survey
}

\author{
Thierry Bouwmans
}

\section{To cite this version:}

Thierry Bouwmans. Recent Advanced Statistical Background Modeling for Foreground Detection - A Systematic Survey. Recent Patents on Computer Science, 2011, 4 (3), pp.147-176. hal-00644746

HAL Id: hal-00644746

https://hal.science/hal-00644746

Submitted on 19 Nov 2015

HAL is a multi-disciplinary open access archive for the deposit and dissemination of scientific research documents, whether they are published or not. The documents may come from teaching and research institutions in France or abroad, or from public or private research centers.
L'archive ouverte pluridisciplinaire $\mathbf{H A L}$, est destinée au dépôt et à la diffusion de documents scientifiques de niveau recherche, publiés ou non, émanant des établissements d'enseignement et de recherche français ou étrangers, des laboratoires publics ou privés. 


\title{
Recent Advanced Statistical Background Modeling for Foreground Detection - A Systematic Survey
}

\author{
Thierry Bouwmans \\ Laboratoire MIA, Université de La Rochelle, Avenue M. Crépeau, 17000 La Rochelle, France \\ Tel Phone: (33) 05.46.45.72.02 \\ Email address: tbouwman@univ-lr.fr \\ Fax number: (33) 05.46.45.82.40
}

Short Running Title: Advanced Background Modeling: A Systematic Survey

\begin{abstract}
Background modeling is currently used to detect moving objects in video acquired from static cameras. Numerous statistical methods have been developed over the recent years. The aim of this paper is firstly to provide an extended and updated survey of the recent researches and patents which concern statistical background modeling and secondly to achieve a comparative evaluation. For this, we firstly classified the statistical methods in term of category. Then, the original methods are reminded and discussed following the challenges met in video sequences. We classified their respective improvements in term of strategies used. Furthermore, we discussed them in term of the critical situations they claim to handle. Finally, we conclude with several promising directions for future research. The survey also discussed relevant patents.
\end{abstract}

Keywords: Background modeling, Kernel Density Estimation, Mixture of Gaussians, Single Gaussian, Subspace Learning

\section{INTRODUCTION}

Different applications such as video surveillance [1], optical motion capture [2-4] and multimedia [5-7] need firstly to model the background and then to detect the moving objects. One way to obtain the background is to acquire a background image which doesn't include any moving object but in some environment the background is not available. Furthermore, it can always be changed under critical situations like illumination changes, objects being introduced or removed from the scene. To take into account these problems, many background modeling methods have been developed $[8,9]$ and these methods can be classified in the following categories:

- Basic Background Modeling: In this case, the background is modeled using the average [10] or the median [11] or the histogram analysis over time [12].

- Statistical Background Modeling: The background is modeled using a single Gaussian [13] or a Mixture of Gaussians [14] or a Kernel Density Estimation [15]. Statistical variables are used to classify the pixels as foreground or background.

- Fuzzy Background Modeling: The background is modeled using a fuzzy running average [16] or Type-2 fuzzy mixture of Gaussians [17]. Foreground detection is made using the Sugeno integral [18] or the Choquet integral [19]. The foreground detection can be performed by fuzzy inferences [335].

*Address correspondence to these authors at the Laboratory de Mathematics Image and Applications (LMIA), Pôle Science, Université de La Rochelle, 17000 La Rochelle, France; E-mail: tbouwman@univ-lr.fr
- Background Clustering: The background model supposes that each pixel in the frame can be represented temporally by clusters. Incoming pixels are matched against the corresponding cluster group and are classified according to whether the matching cluster is considered part of the background. The clustering approach consists in using K-mean algorithm [361] or using Codebook [362].

- Neural Network Background Modeling: The background is represented by mean of the weights of a neural network suitably trained on $\mathrm{N}$ clean frames. The network learns how to classify each pixel as background or foreground [332][333].

- Wavelet Background Modeling: The background model is defined in the temporal domain, utilizing the coefficients of discrete wavelet transform (DWT) [336].

- Background Estimation: The background is estimated using a filter. Any pixel of the current image that deviates significantly from its predicted value is declared foreground. This filter may be a Wiener filter [20], a Kalman filter [21] or a Tchebychev filter [22].

Table 1 shows an overview of this classification. The first column indicates the category and the second column the name of each method. The number of papers counted for each method is indicated in the parenthesis. The third column gives the name of the authors who have made the main publication for the corresponding method and the date of the related publication. Other classifications can be found in term of prediction [23], recursion [1], adaptation [24], or modality [25]. 
Table 1. Background Modeling Methods: An Overview

\begin{tabular}{|c|c|c|}
\hline Category & Methods & Authors - Dates \\
\hline Basic Background Modeling & $\begin{array}{l}\text { Mean (11) } \\
\text { Median (13) } \\
\text { Histogram over time (13) }\end{array}$ & $\begin{array}{l}\text { Lee } \text { et al. (2002) [10] } \\
\text { Mac Farlane } \text { et al. }(1995) \text { [11] } \\
\text { Zheng et al. (2006) [12] }\end{array}$ \\
\hline Statistical Background Modeling & $\begin{array}{l}\text { Single Gaussian (33) } \\
\text { Mixture of Gaussians (217) } \\
\text { Kernel Density Estimation (5) }\end{array}$ & $\begin{array}{l}\text { Wren et al. (1997) [13] } \\
\text { Stauffer and Grimson (1999) [14] } \\
\text { Elgammal et al. (2000) [15] }\end{array}$ \\
\hline Fuzzy Background Modeling & $\begin{array}{l}\text { Fuzzy Running Average (5) } \\
\text { Type-2 Fuzzy Mixture of Gaussians (3) }\end{array}$ & $\begin{array}{l}\text { Sigari et al. (2008) [16] } \\
\text { El Baf et al. (2008) [17] }\end{array}$ \\
\hline Background Clustering & $\begin{array}{l}\text { K-Means (11) } \\
\text { Codebook (35) }\end{array}$ & $\begin{array}{l}\text { Butler et al. (2003) [361] } \\
\text { Kim et al. (2005) [362] }\end{array}$ \\
\hline Neural Network Background Modeling & $\begin{array}{l}\text { General Regression Neural Network (1) } \\
\text { Self Organizing Neural Network (9) }\end{array}$ & $\begin{array}{l}\text { Culibrk et al. (2006) [332] } \\
\text { Maddalena and Petrosino (2007) [333] }\end{array}$ \\
\hline Wavelet Background Modeling & Discrete Wavelet Transform & Biswas et al. [336] \\
\hline Background Estimation & $\begin{array}{l}\text { Wiener Filter (1) } \\
\text { Kalman Filter (19) } \\
\text { Tchebychev Filter (3) }\end{array}$ & $\begin{array}{l}\text { Toyama et al. (1999) [20] } \\
\text { Messelodi et al. }(2005)[21] \\
\text { Chang et al. }(2004)[22]\end{array}$ \\
\hline
\end{tabular}

All these modeling approaches are used in background subtraction context which presents the following steps and issues: background modeling, background initialization, background maintenance, foreground detection, choice of the feature size (pixel, a block or a cluster), choice of the feature type (color features, edge features, stereo features, motion features and texture features). Developing a background subtraction method, all these choices determine the robustness of the method to the critical situations met in video sequence $[5,20]$ : Noise image due to a poor quality image source (NI), Camera jitter (CJ), Camera automatic adjustments (CA), Time of the day (TD), Light switch (LS), Bootstrapping (B), Camouflage (C), Foreground aperture (FA), Moved background objects (MO), Inserted background (IB), Waking foreground object (WFO), Sleeping foreground object (SFO) and Shadows (S). The main difficulties come from the dynamic backgrounds and illumination changes:

- Dynamic backgrounds often appear in outdoor scenes. Fig. (1). presents four typical examples: Camera jitter, waving trees, water rippling and water surface. The left column shows the original images and the right the foreground mask obtained by the MOG [14]. In each case, there is a big amount of false detections.

- Illumination changes appear in indoor and outdoor scenes. Fig. (2). shows an indoor scene in which we can observe a gradual illumination change. This causes false detections in several parts of the foreground mask obtained by the MOG [14]. Fig. (3). illustrates the case of sudden illumination change due to a light on/off. Every pixel in the images is affected by this change which generates a large amount of false detections (see Fig. 3c).
Fig. (1). The first column presents original scenes containing dynamic backgrounds. The second column shows the foreground masks obtained by the MOG [14].
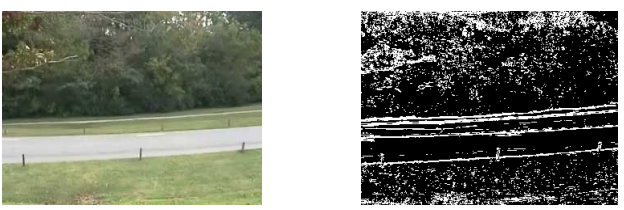

a) Sequence Camera jitter from [229]
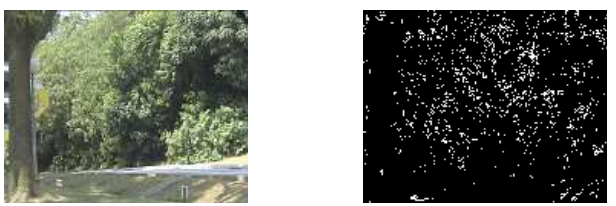

b) Sequence Campus from [34]
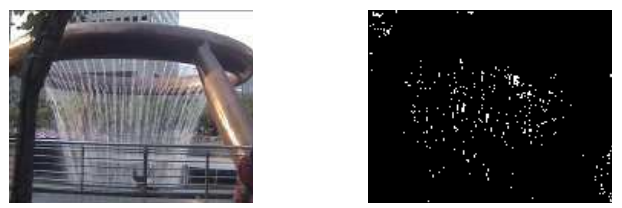

c) Sequence Water rippling from [34]
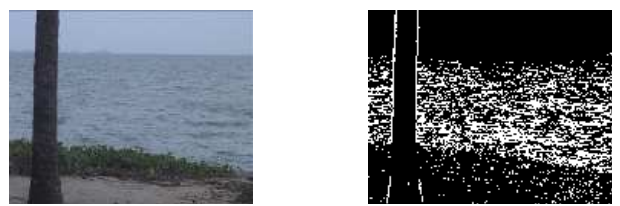

d) Sequence Water surface from [34] 
Fig. (2). From left to right: The first image presents an indoor scene with low illumination. The second image presents the same scene with a moderate illumination while the third image shows the scene with a high illumination. The fourth image shows the foreground mask obtained with MOG [14]. This sequence called "Time of Day" comes from the Wallflower dataset [20].

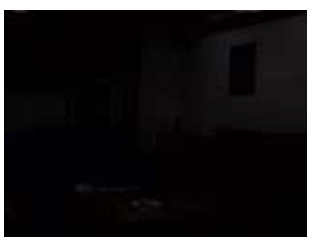

a) Low

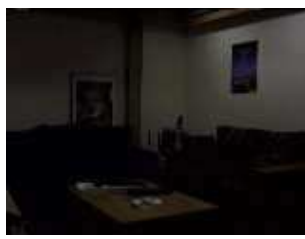

b) Moderate

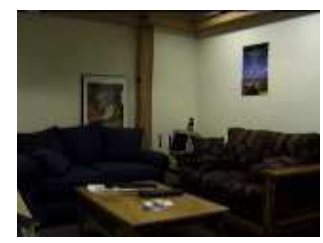

c) High

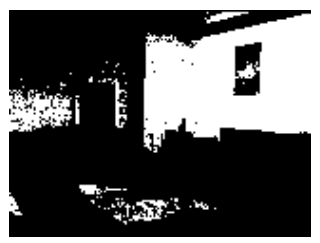

d) Foreground mask

Fig. (3). From left to right: The first image presents an indoor scene with light-on. The second image shows the same scene with light-off. The third image shows the foreground mask obtained with MOG [14]. This sequence called "Light Switch" comes from the Wallflower dataset [20].

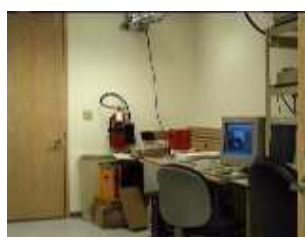

a) Light-on

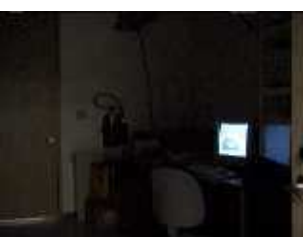

b) Light-off

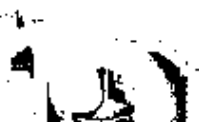

c) Foreground mask
Different datasets benchmarks are available [26-31] to evaluate the robustness of the background subtraction methods against these critical situations which have different spatial and temporal characteristics which must be take into account to obtain a good segmentation. This challenge must be made in the context of real-time application which runs on common PC and so two constraints are introduced: less computation time (CT) and less memory requirement (MR) as possible. The performance is evaluated using the ROC analysis [32] or the PDR Analysis [33] or the similarity measure [34]. Others performance evaluation methods are proposed and compared in [35, 36]. Reading the literature, two main remarks can be made: (1) The most frequently used models are the statistical ones due to their robustness to the critical situations. (2) There are many recent developments regarding statistical models as can be seen for the MOG model with the acronyms found like GMM [37], TLGMM [38], STGMM [39], SKMGM [40], TAPPMOG [41] and S-TAPPMOG [42]. The objective is then to categorize the statistical models in one paper and classify their recent improvements following the strategies used. We also discuss them following the challenges met in video sequences and evaluate some of them in term of false alarms using the Wallflower dataset [20].

This paper is an extended and updated paper of the surveys on Mixture of Gaussians for background modeling [48] and Subspace Learning for background modeling [334].

The rest of this paper is organized as follows: In Section 2, we firstly provide a background on the statistical background models and a classification of these models. In Section 3, we survey the first generation models and their respective improvements. In Section 4, we classified the second generation models. In Section 5, the third generation models are reviewed. In Section 6, we firstly investigated the performance in term of robustness on dynamic backgrounds and illumination changes and secondly in terms on per-pixel complexity. Then, a comparative evaluation is provided in Section 7. Finally, conclusion and future developments are given.

\section{STATISTICAL BACKGROUND MODELING: AN OVERVIEW}

The statistical tools provide a good framework to model the background and so many methods have been developed. We classified them in term of category as follows:

First category: The first way to represent statistically the background is to assume that the history over time of intensity values of a pixel can be modeled by a single Gaussian (SG) [13]. However, a unimodal model cannot handle dynamic backgrounds when there are waving trees, water rippling or moving algae. To solve this problem, the Mixture of Gaussians (MOG) has been used to model dynamic backgrounds [14]. This model has some disadvantages. Background having fast variations cannot be accurately modeled with just a few Gaussians (usually 3 to 5), causing problems for sensitive detection. So, a non-parametric technique was developed for estimating background probabilities at each pixel from many recent samples over time using Kernel density estimation (KDE) [15] but it is time consuming. In [165], Subspace Learning using Principal Component Analysis (SL-PCA) is applied on N images to construct a background model, which is represented by the mean image and the projection matrix comprising the first $\mathrm{p}$ significant eigenvectors of PCA. In this way, foreground segmentation is accomplished by computing the difference between the input image and its reconstruction. 
Table 2.Advanced Statistical Background Modeling: An Overview

\begin{tabular}{|c|c|c|}
\hline Category & Methods & Authors - Dates \\
\hline First Category & $\begin{array}{l}\text { Single Gaussian (SG) (33) } \\
\text { Mixture of Gaussians (MOG) (217) } \\
\text { Kernel Density Estimation (KDE) (55) } \\
\text { Principal Components Analysis (SL-PCA) (25) }\end{array}$ & $\begin{array}{l}\text { Wren et al. }(1997)[13] \\
\text { Stauffer and Grimson (1999) [14] } \\
\text { Elgammal } \text { et al. }(2000)[15] \\
\text { Oliver } \text { et al. } \text { et al. }(1999) \text { [165] }\end{array}$ \\
\hline Second Category & $\begin{array}{l}\text { Support Vector Machine (SVM) (9) } \\
\text { Support Vector Regression (SVR) (3) } \\
\text { Support Vector Data Description (SVDD) (6) }\end{array}$ & $\begin{array}{l}\text { Lin et al. (2002) [180] } \\
\text { Wang et al. (2006) [183] } \\
\text { Tavakkoli et al. }(2006)[186]\end{array}$ \\
\hline Third Category & $\begin{array}{l}\text { Single General Gaussian (SGG) (3) } \\
\text { Mixture of General Gaussians (MOGG) (3) } \\
\text { Independent Component Analysis (SL-ICA) (3) } \\
\text { Incremental Non Negative Matrix Factorization (SL-INMF) (3) } \\
\text { Incremental Rank- }\left(\mathrm{R}_{1}, \mathrm{R}_{2}, \mathrm{R}_{3}\right) \text { Tensor (SL-IRT) (2) }\end{array}$ & $\begin{array}{l}\text { Kim et al. }(2007)[190] \\
\text { Allili et al. }(2007)[194] \\
\text { Yamazaki et al. }(2006)[198] \\
\text { Bucak et al. }(2007)[202] \\
\text { Li et al. }(2008)[204]\end{array}$ \\
\hline
\end{tabular}

- Second category: This second category uses support vector models. The objective is different following the models used. Lin et al. [180] used a SVM algorithm to initialize the background in outdoor scene. Wang et al. $[183,184]$ modeled the background by using SVR in the case of traffic surveillance scene where illumination changes (TD) appear. Tavakkoli et al. [186-189] applied SVDD to deal with dynamic backgrounds (MB).

- Third category: These models generalize the first generation model as the single general Gaussian (SGG) [190-192], the mixture of general Gaussians (MOGG) [193-195] and subspace learning using Independent Component Analysis (SL-ICA) [198, 200], Incremental Non-negative Matrix Factorization (SL-INMF) [202, 203] or Incremental Rank- $\left(\mathrm{R}_{1}, \mathrm{R}_{2}, \mathrm{R}_{3}\right)$ Tensor (SL-IRT) $[204,205]$. The single general Gaussian (SGG) alleviates the constraint of a strict Gaussian and then shows better performance in the case of illumination changes (TD) and shadow (S). The MOGG have been developed to be more robust to dynamic backgrounds (MB). Subspace learning methods are more robust to illumination changes (LS).

Table 2 shows an overview of the statistical background modeling. The first column indicates the generation and the second column the name of each method. Their corresponding acronym is indicated in the first parenthesis and the number of papers counted for each method in the second parenthesis. The third column gives the name of the authors who have made the main publication for the corresponding method and the date of the related publication. We can see that the MOG with 217 papers is the most modified and improved because it is the most used due to a good compromise between robustness.

In the following sections, we remind the original methods for each generation and we have classify their related improvements in the following way: intrinsic improvements which concern the modification made in the initialization, the maintenance and the foreground detection steps, and extrinsic improvements which consist in using external tools to perform the results.

\section{FIRST CATEGORY}

\subsection{Single Gaussian (SG)}

Wren et al. [13] proposed to model the background independently at each pixel location $(i, j)$. The model is based on ideally fitting a Gaussian probability density function on the last $\mathrm{n}$ pixel's values. In order to avoid fitting the pdf from scratch at each new frame time $t+1$, the mean and the variance are updated as follows:

$$
\begin{gathered}
\mu_{t+1}=(1-\alpha) \mu_{t}+\alpha \cdot X_{t+1} \\
\sigma_{t+1}^{2}=(1-\alpha) \sigma_{t}^{2}+\alpha\left(X_{t+1}-\mu_{t+1}\right)\left(X_{t+1}-\mu_{t+1}\right)^{T}
\end{gathered}
$$

where $X_{t+1}$ is the pixel's current value, $\mu_{t}$ is the previous average, $\sigma_{t}$ is the previous variance and $\alpha$ is the learning rate. The foreground detection is made as follows:

if $\left|\mu_{t+1}-X_{t+1}\right|<T$, the pixel is classified as background otherwise the pixel is classified as foreground.

Improvements: Medioni et al. [43] operated in the HueSaturation-Value (HSV) color space instead of the RGB one. The advantage is that the HSV color model is more robust to gradual illumination changes (TD) because it separates the intensity and chromatic information. Furthermore, HSV permits to eliminate partially camouflage. Zhao et al. [44] used HSV too remarking that the respective distributions of $\mathrm{H}$ and $\mathrm{S}$ vary naturally a lot and that the distribution of $\mathrm{V}$ is the most stable. So, the component $\mathrm{H}$ and $\mathrm{S}$ are only used when they are stable. Results [44] show better performance in presence of gradual illumination changes (TD) and shadows (S).

Discussion: The single Gaussian (SG) is suited for indoor scenes where there are moderate illumination changes. 


\subsection{Mixture of Gaussians (MOG)}

In the context of a traffic surveillance system, Friedman and Russel [45] proposed to model each background pixel using a mixture of three Gaussians corresponding to road, vehicle and shadows. This model is initialized using an EM algorithm. Then, the Gaussians are manually labeled in a heuristic manner as follows: the darkest component is labeled as shadow; in the remaining two components, the one with the largest variance is labeled as vehicle and the other one as road. This remains fixed for all the process giving lack of adaptation to changes over time. For the foreground detection, each pixel is compared with each Gaussian and is classified according to it corresponding Gaussian. The maintenance is made using an incremental EM algorithm for real time consideration. Stauffer and Grimson [14] generalized this idea by modeling the recent history of the color features of each pixel $\left\{X_{1}, \ldots, X_{t}\right\}$ by a mixture of $\mathrm{K}$ Gaussians. We remind below the algorithm.

\section{Principle}

First, each pixel is characterized by its intensity in the RGB color space. Then, the probability of observing the current pixel value is considered given by the following formula in the multidimensional case:

$$
P\left(X_{t}\right)=\sum_{i=1}^{K} \omega_{i, t} . \eta\left(X_{t}, \mu_{i, t}, \sum_{i, t}\right)
$$

where the parameters are $\mathrm{K}$ is the number of distributions, $\omega_{i, t}$ is a weight associated to the $\mathrm{i}^{\text {th }}$ Gaussian at time $\mathrm{t}$ with mean $\mu_{i, t}$ and standard deviation $\sum_{i, t} . \eta$ is a Gaussian probability density function:

$$
\eta\left(X_{t}, \mu, \Sigma\right)=\frac{1}{(2 \pi)^{n / 2}|\Sigma|^{1 / 2}} e^{-\frac{1}{2}\left(X_{t}-\mu\right) \Sigma^{-1}\left(X_{t}-\mu\right)}
$$

For computational reasons, Stauffer and Grimson [14] assumed that the RGB color components are independent and have the same variances. So, the covariance matrix is of the form:

$$
\Sigma_{i, t}=\sigma_{i, t}^{2} I
$$

So, each pixel is characterized by a mixture of K Gaussians. Once the background model is defined, the different parameters of the mixture of Gaussians must be initialized. The parameters of the MOG's model are the number of Gaussians $\mathrm{K}$, the weight $\omega_{i, t}$ associated to the $\mathrm{i}^{\text {th }}$ Gaussian at time $\mathrm{t}$, the mean $\mu_{i, t}$ and the covariance matrix $\sum_{i, t}$.

\section{Remarks:}

- $\quad \mathrm{K}$ determined the multimodality of the background and by the available memory and computational power. Stauffer and Grimson [14] proposed to set K from 3 to 5.

- The initialization of the weight, the mean and the covariance matrix is made using an EM algorithm. Stauffer and Grimson [14] used the K-mean algorithm for real time consideration.

Once the parameters initialization is made, a first foreground detection can be made and then the parameters are updated. Firstly, Stauffer and Grimson [14] used as criterion the ratio $r_{j}=\omega_{j} / \sigma_{j}$ and ordered the $\mathrm{K}$ Gaussians following this ratio. This ordering supposes that a background pixel corresponds to a high weight with a weak variance due to the fact that the background is more present than moving objects and that its value is practically constant. The first B Gaussian distributions which exceed certain threshold $T$ are retained for a background distribution:

$$
B=\arg \min _{b}\left(\sum_{i=1}^{b} \omega_{i, t}>T\right)
$$

The other distributions are considered to represent a foreground distribution. Then, when the new frame incomes at times $t+1$, a match test is made for each pixel. A pixel matches a Gaussian distribution if:

$\operatorname{sqrt}\left(\left(X_{t+1}-\mu_{i, t}\right)^{T} \cdot \sum_{i, t}^{-1} \cdot\left(X_{t+1}-\mu_{i, t}\right)\right)<k \sigma_{i, t}$ (5) where

$k$ is a constant threshold equal to 2.5 . Then, two cases can occur:

- Case 1: A match is found with one of the $\mathrm{K}$ Gaussians. In this case, if the Gaussian distribution is identified as a background one, the pixel is classified as background else the pixel is classified as foreground.

- Case 2: No match is found with any of the $\mathrm{K}$ Gaussians. In this case, the pixel is classified as foreground.

At this step, a binary mask is obtained. Then, to make the next foreground detection, the parameters must be updated. Using the match test (5), two cases can occur like in the foreground detection:

Case 1: A match is found with one of the $\mathrm{K}$ Gaussians.

For the matched component, the update is done as follows:

$$
\omega_{i, t+1}=(1-\alpha) \omega_{i, t}+\alpha
$$

where $\alpha$ is a constant learning rate.

$$
\mu_{i, t+1}=(1-\rho) \mu_{i, t}+\rho \cdot X_{t+1}
$$




$$
\begin{gathered}
\sigma_{i, t+1}^{2}=(1-\rho) \sigma_{i, t}^{2}+\rho\left(X_{t+1}-\mu_{i, t+1}\right) \cdot\left(X_{t+1}-\mu_{i, t+1}\right)^{T} \\
\text { where } \rho=\alpha \cdot \eta\left(X_{t+1}, \mu_{i}, \sum_{i}\right)
\end{gathered}
$$

- For the unmatched components, $\mu$ and $\sum$ are unchanged, only the weight is replaced by:

$$
\omega_{j, t+1}=(1-\alpha) \omega_{j, t}
$$

Case 2: No match is found with any of the K Gaussians. In this case, the least probable distribution $\mathrm{k}$ is replaced with a new one with parameters:

$$
\begin{aligned}
& \omega_{k, t+1}=\text { Low Prior Weight } \\
& \mu_{k, t+1}=X_{t+1} \\
& \sigma_{k, t+1}^{2}=\text { Large Initial Variance }
\end{aligned}
$$

Once the parameters maintenance is made, foreground detection can be made and so on. Complete studies on the signification and the setting of the parameters can be found in $[46,47][218][289]$.

Improvements: The original MOG presents several advantages. Indeed, it can work without having to store an important set of input data in the running process. The multimodality of the model allows dealing with multimodal backgrounds and gradual illumination changes. Despite it, this model present some disadvantages: the number of Gaussians must be predetermined, the need for good initializations, the dependence of the results on the true distribution law which can be non-Gaussian and slow recovery from failures. Others limitations are the needs for a series of training frames absent of moving objects and the amount of memory required in this step. To alleviate these limitations, numerous improvements (217 papers) have been proposed over the recent years. All the developed improvements can be classified following the strategies and a complete survey over 100 papers in the period 1999-2007 can be found in [48]. We have summarized and updated them in the following classification:

Intrinsic improvements: These strategies (Table 3) consist to be more rigorous in the statistical sense or to introduce spatial and/or temporal constraint in the different step of the model. For example, some authors [49-53] propose to determine automatically and dynamically the number of Gaussians to be more robust to dynamic backgrounds. Other approaches use another algorithm for the initialization $[54,55]$ and allow presence of foreground objects in the training sequence $[56,57,58]$. For the maintenance, the learning rates are better set $[66,67]$ or adapt over time [60-62, 68-78]. For the foreground detection, the improvement found in the literature are made using a different measure for the matching test [53, 79-82], using a Pixel Persistence Map
(PPM) [75, 76, 83], using the probabilities [84, 85], using a foreground model $[61,63,86]$, using some matching tests $[39,60]$ and using the most dominant background model [87, 88, 89]. For the feature size, block wise $[90,91]$ or cluster wise [92] approaches are more robust than the pixel one. For the feature type, several features are used instead of the RGB space like different color features [93-99], edge features [100, 101], texture features [102], stereo feature [103, 104], spatial features [105], motion features [40] and video features [106]. Zheng et al. [267, 268] combined multiple features such as brightness, chromaticity and neighborhood information. Recent patents concern block wise approaches [352], texture features [353], motion features [354] and spatial features [355]. An overview of the different features used in the literature is shown in Table 5.

- Extrinsic improvements: Another way to improve the efficiency and robustness of the original GMM consist in using external strategies (Table 4). Some authors used Markov Random Fields [107-109], hierarchical approaches [110-113], multi-level approaches [100, 114-118], multiple backgrounds [119, 121], graph cuts [81], multi-layer approaches [122, 123], tracking feedback $[128,129]$ or specific post-processing [130131]. Recent patents concern graph cuts approaches [3576, 357].

Reducing the computation time: All the intrinsic and extrinsic improvements concern the quality of the foreground detection but there is another manner to improve the original MOG which consists in reducing the computation time. It achieved by using region of interest [132] [287], by using a variable adaption rate [133], by switching the background model [134] [271], by using space sampling strategies [135][216][238][272] or by using hardware implementation [136, 137] [271].

Enhancing the foreground detection: All the previous improvements concern directly the original MOG and the foreground detection results only from it. Another way to improve this method is to enhance the results of the foreground detection by using cooperation with another segmentation method. It achieved by cooperation with a statistical background disturbance technique [138], with color segmentation [139], and with a region based motion detection [140]. Other authors used a cooperation with optical flow [217], block matching [247-248], predictive models [249], texture models [251][303], consecutive frame difference [258][261-262][279-280][282] and basic background subtraction [304-305][330]. A recent patent concern the cooperation with histogram statistics [358].

Table $\mathbf{6}$ and Table $\mathbf{7}$ show respectively an overview of the critical situations and the real-time constraints for the different MOG versions that can tackle them better than the original one. 
Table 3. Intrinsic improvements of the MOG

\begin{tabular}{|c|c|c|}
\hline Background Step & Parameters & Authors - References \\
\hline \multirow[t]{2}{*}{$\begin{array}{l}\text { Background } \\
\text { Initialization }\end{array}$} & Variable K & $\begin{array}{l}\text { Zivkovic [49], Cheng et al. [50], Shimada et al. [51], Tan et al. [52], Carminati et } \\
\text { al. [53], Klare and Sarka [230], Shimada et al. [237], Shahid et al. [240], Singh } \\
\text { and Mitra [248], Wang et al. [278], Huang et al. [288], Wang et al. [307], Zhou et } \\
\text { al. [317] }\end{array}$ \\
\hline & Variables $\mu, \sigma, \omega$ & $\begin{array}{l}\text { Another algorithm: Morellas et al. [54], Lee [55], Ju et al.. [241], Singh et al. } \\
\text { [245], Singh et al. [246], Wang and Dai [252], Hu et al.. [259], Guo et al. [270], } \\
\text { Molin [285], Qin et al. [286], Li et al. [315], Wang and Miller [331] } \\
\text { Allowing presence of moving objects: Zhang et al. [56], Amintoosi et al. [57], } \\
\text { Lepisk [58], Lee } \text { et al. [273], Wang et al. } \text { [307] }\end{array}$ \\
\hline \multirow[t]{3}{*}{$\begin{array}{l}\text { Background } \\
\text { Maintenance }\end{array}$} & Variable $\mathrm{K}$ & $\begin{array}{l}\text { Zivkovic [49], Cheng et al. [50], Shimada et al. [51], Tan et al. [52], Klare and } \\
\text { Sarka [230], Shimada et al. [237], Singh and Mitra [248], Wang et al. [278], } \\
\text { Zhou et al. [317] }\end{array}$ \\
\hline & Variables $\mu, \sigma, \omega$ & $\begin{array}{l}\text { Maintenance rules: Han and Li [59], Park and Buyn [266] } \\
\text { Maintenance mechanisms: Zhang et al. [56], Wang and Suter [60], Lindstrom et } \\
\text { al. [61], Li et al. [269], Lee } \text { et al. [273] } \\
\text { Selective maintenance: Stauffer and Grimson [62], Landabaso and Pardas [63], } \\
\text { Park et al. [64], Mittal and Huttenlocher [65], Salas et al. [215], Wang and Dai } \\
\text { [252], Hu et al. [259], Li et al. [265], Liu and Zhang [276], Yu et al. [290] }\end{array}$ \\
\hline & Learning rates $\alpha, \rho$ & $\begin{array}{l}\text { Better settings: Zang and Klette [66], White and Shah [67] } \\
\text { Adaptive learning rates: Wang and Suter [60], Lindstrom et al. [61], Stauffer and } \\
\text { Grimson [62], KaewTraKulPong and Bowden [68-70] Lee[71], Harville } \text { et al. } \\
\text { [72], Porikli [73], Liu et al. [74], Pnevmatikakis } \text { et al. [75, 76], Power } \text { et al. [77], } \\
\text { Leotta } \text { et al. } \text { [78], Sheng and Cui [272], Quast } \text { et al. [284], Molin [285], Qin } \text { et al. } \\
\text { [286], Shah et al. [298], Kan et al. [302], Quast } \text { et al. [308], Lin et al. [309], Bin } \\
\text { and Liu [320], Zhao and He [322], Li et al. [323] }\end{array}$ \\
\hline \multirow[t]{7}{*}{$\begin{array}{l}\text { Foreground } \\
\text { Detection }\end{array}$} & $\begin{array}{l}\text { Different measure for the } \\
\text { matching test }\end{array}$ & $\begin{array}{l}\text { Carminati et al. [53], Ren at al. [79], Lee [80], Sun [81], Morellas et al. [82], } \\
\text { Xuehua et al. [261], Rui et al. [262] }\end{array}$ \\
\hline & $\begin{array}{l}\text { Pixel Persistence Map } \\
(\mathrm{PPM})\end{array}$ & Pnevmatikakis et al. $[75,76]$, Landabaso and Pardas [83] \\
\hline & Probabilities & Yang and Hsu [84], Lee [85], Lien et al. [251], Zhang and Zhou [21] \\
\hline & Foreground model & $\begin{array}{l}\text { Lindstrom et al. [61], Landabaso et al. [63], Withagen et al. [86], Landabaso et } \\
\text { al. [263], Feldman et al. [313], Feldman [314], Tian and Wang [318] }\end{array}$ \\
\hline & Some matching tests & Zhang et al. [39], Wang and Suter [60] \\
\hline & Fusion rules & Lien et al. [251] \\
\hline & Most dominant background & Haque et al. $[87,88,89]$ \\
\hline
\end{tabular}

Table 4.Extrinsic improvements of the MOG

\begin{tabular}{|c|c|}
\hline Methods & Authors - References \\
\hline Markov Random Fields & $\begin{array}{l}\text { Kumar and Sengupta [107], Zhou and Zhang [108], Schindler and Wang [109], Landabaso } \\
\text { et al. [263], Li et al [291], Dickinson et al. [316], Zhang and Zhou [327], Wang et al. [328] }\end{array}$ \\
\hline Hierarchical approaches & $\begin{array}{l}\text { Sun and Yuan [110], Park et al. [111], Chen et al. [112], Zhou et al. [113], Zhong et al. } \\
\text { [242], Zhong et al. [264], Li et al. [265] }\end{array}$ \\
\hline Multi-level approaches & $\begin{array}{l}\text { Javed et al. [100], Zang and Klette [114], Zhong et al. [115], Cristani et al. [116-118], } \\
\text { Yang et al. [325] }\end{array}$ \\
\hline Multiple backgrounds & Su and $\mathrm{Hu}[119,120]$, Porikli [121], Qi et al. [310], Qi et al. [311] \\
\hline Graph cuts & Sun [81], Chang and Hsu [257], Li et al. [269], Li et al. [291] \\
\hline Multi-layer approaches & Yang et al. [122], Porikli and Tuzel [123], Park and Buyn [266], Huang and Wu [292] \\
\hline Features-Cameras strategies & $\mathrm{Xu}$ and Ellis [124], Nadimi and Bhanu [125, 126], Conaire et al. [127] \\
\hline Tracking feedback & $\begin{array}{l}\text { Harville [128], Taycher et al. [129], Wang et al. [275], He et al. [301], Yuan et al. [344], } \\
\text { Shao et al. [326] }\end{array}$ \\
\hline Post-processing & Turdu and Erdogan [130], Parks and Fels [131], Fazli et al. [306] \\
\hline
\end{tabular}


Table 5. Features improvements of the MOG

\begin{tabular}{|c|c|c|}
\hline & Size/Type & Authors - References \\
\hline \multirow[t]{2}{*}{ Feature Size } & Block & $\begin{array}{l}\text { Fang et al. [90], Pokrajac and Latecki [91], Wang et al. [275], Zhong et al. } \\
\text { [281], Zhang et al. [294], Wang et al. [329] }\end{array}$ \\
\hline & Cluster & Bhaskar et al. [92], Cai et al. [243] \\
\hline \multirow[t]{11}{*}{ Feature Type } & $\begin{array}{l}\text { Color features } \\
\text { Normalized RGB } \\
\text { YUV } \\
\text { HSV } \\
\text { HSI } \\
\text { Luv } \\
\text { Improved HLS } \\
\text { YCrCb }\end{array}$ & $\begin{array}{l}\text { Stijman } \text { et al. [93], Xu et Ellis [94] } \\
\text { Harville et al.[72], Sun [81], Fang et al. [90], Guo et al. [270], Feldman et al. } \\
\text { [313], Feldman [314] } \\
\text { Sun [81], Xuehua } \text { et al. [261], Rui } \text { et al. [262], Wang and Tang [274] } \\
\text { Wang and Wu [95] } \\
\text { Yang and Hsu [96] } \\
\text { Setiawan } \text { et al. [97] } \\
\text { Kristensen } \text { et al. [98], Ribeiro } \text { et al. [99] }\end{array}$ \\
\hline & Edge features & Javed et al. [100], Jain et al. [101], Klare and Sarka [203], Li et al. [253] \\
\hline & Texture features & Tian and Hampapur [102], Shimada and Taniguchi [250], Huang et al. [255] \\
\hline & $\begin{array}{c}\text { Stereo features } \\
\text { Disparity } \\
\text { Depth }\end{array}$ & $\begin{array}{l}\text { Gordon et al. [103] } \\
\text { Harville et al. [72], Silvestre [104] }\end{array}$ \\
\hline & Spatial features & $\begin{array}{l}\text { Yang and Hsu [84], Dickinson et al. [105], Klare and Sarka [230], Wei et al. } \\
\text { [231] }\end{array}$ \\
\hline & Motion features & Tang et al. [40] \\
\hline & Phase features & Xue et al. [312] \\
\hline & Video features & Wang et al. [106], Wang et al. [239] \\
\hline & Entropy features & Park et al. [295], Park et al. [296] \\
\hline & Bayer features & Suhr et al. [297] \\
\hline & HOG features & Fabian [299], Hu et al. [300] \\
\hline
\end{tabular}

Table 6. Challenges and MOG Versions

\begin{tabular}{|c|c|}
\hline Critical Situations & Authors - References \\
\hline CS 1 - Noise Image & X Xu [221], Teixeira et al. [222], Li et al. [265] \\
\hline CS 2-1 - Camera jitter & $\begin{array}{l}\text { Campbell-West et al. [219], Xu [221], Achkar } \\
\text { and Amer [223], Rao et al. [224], Li et al. [265] }\end{array}$ \\
\hline CS 2-2 - Camera Adjustements & Zen and Lai [225], Molin [285] \\
\hline CS 3 - Gradual Illumination Changes & $\begin{array}{l}\text { Tian et al. [234], Huang et al. [254], Wang et al. } \\
\text { [277], Baloch [283], Huang et al. [288], Lin et } \\
\text { al. [309] }\end{array}$ \\
\hline CS 4 - Sudden Illumination Changes & $\begin{array}{l}\text { Tian et al. [234], Li et al. [253], Baloch [283], } \\
\text { Lin } \text { et al. [309], Xue et al. [312], Li et al. [323] }\end{array}$ \\
\hline CS 5-1 - Bootstrapping during initialization & Gao et al. [220] \\
\hline CS 5-2 - Bootstrapping during maintenance & Lindstrom et al. [61] \\
\hline CS 6 - Camouflage & Guo et al. [270] \\
\hline CS7 - Foreground Aperture & Utasi and Czúni [226] \\
\hline CS 8 - Moved background objects & Teixeira et al. [222] \\
\hline CS 9 - Inserted background objects & Teixeira et al. [222] \\
\hline CS 10 - Multimodal background & Dalley et al. [227], Li et al. [265] \\
\hline CS 11 - Waking foreground object & $\mathrm{Su}$ and $\mathrm{Hu}[119], \mathrm{Hu}$ and $\mathrm{Su}[120]$ \\
\hline CS 12 - Sleeping foreground objects & $\begin{array}{l}\text { Cheng et al. [229], Cai et al. [256], Hu et al. } \\
\text { [259] }\end{array}$ \\
\hline CS 13 - Shadows Detection & $\begin{array}{l}\text { Xu [221], Huang and Chen [232], Zhang et al. } \\
\text { [233], Tian et al. [234], Izadi et al. [235], } \\
\text { Rahman [236], Chen et al. [260], Landabaso et } \\
\text { al. [263], Li et al. [265], Quast et al. [284], } \\
\text { Molin [285], Huang et al. [288], Forczmanski } \\
\text { and Seweryn [293], Tian and Wang [318], Li } \\
\text { and Xu [319], Bin and Liu [320], Liu and Bin } \\
\text { [321], Lai } \text { et al. [324], Wang et al. [328] }\end{array}$ \\
\hline
\end{tabular}


Table 7. Real Time Constraints and MOG Versions

\begin{tabular}{|l|l|}
\hline Real-Time Constraints & Authors - References \\
\hline \hline Computation Time & $\begin{array}{l}\text { Cuevas } \text { et al. [228], Chang and Hsu [257], } \\
\text { Krishna } \text { et al. [271] }\end{array}$ \\
\hline Memory Requirement & Krishna et al. [271] \\
\hline
\end{tabular}

Discussion: The Mixture of Gaussians (MOG) is adapted for outdoor scene where there are slow multimodal variations in the backgrounds. For the dynamic backgrounds like camera jitter, waving trees and water rippling, this model causes false detections.

\subsection{Kernel Density Estimation (KDE)}

To deal with dynamic backgrounds like camera jitter, waving trees and water rippling, Elgammal et al. [15] proposed to estimate the probability density function for each pixel using the kernel estimator $K$ for $N$ recent sample of intensity values $\left\{x_{1}, x_{2}, \ldots, x_{N}\right\}$ taken consecutively in a time size window $\mathrm{W}$ as follows:

$$
P\left(x_{t}\right)=\frac{1}{N} \sum_{i=1}^{N} K\left(x_{t}-x_{i}\right)
$$

where $\mathrm{K}()$ is the kernel estimator function which is taken as a Normal Gaussian function $N(0, \Sigma)$. So, the probability density function is determined as follows:

$$
P\left(x_{t}\right)=\frac{1}{N} \sum_{i=1}^{N} \frac{1}{(2 \pi)^{d / 2}|\Sigma|^{1 / 2}} e^{-1 / 2^{*}\left(x_{t}-x_{i}\right)^{T} \Sigma^{-1}\left(x_{t}-x_{i}\right)}
$$

Elgammal et al. [15] assumed that the different color channels are independent with different kernel bandwidths, then the kernel function bandwidth is as follows:

$$
\Sigma=\left(\begin{array}{ccc}
\sigma_{1}^{2} & 0 & 0 \\
0 & \sigma_{2}^{2} & 0 \\
0 & 0 & \sigma_{3}^{2}
\end{array}\right)
$$

So, the probability density function can be written as follows:

$$
P\left(x_{t}\right)=\frac{1}{N} \sum_{i=1}^{N} \prod_{j=1}^{d} \frac{1}{\sqrt{2 \pi \sigma_{j}^{2}}} e^{-1 / 2^{*}\left(x_{t_{j}}-x_{i_{j}}\right)^{T} / \sigma_{j}^{2}}
$$

Elgammal et al. [15] detected the foreground using the probabilities and a threshold $\mathrm{T}$ as follows: If $P\left(x_{t}\right)<T$ then the pixel classified as foreground
else the pixel is classified as background

At this step, a binary mask is obtained. Then, to make the next foreground detection, the parameters must be updated. For this, Elgammal et al. [15] used two background models: a short term one and a long term one. These two models achieve different objectives:

- The short term model adapts quickly to allow very sensitive detection. This model consists of the most recent $\mathrm{N}$ background sample values. The sample is updated using a selective maintenance mechanism, where the decision is based on the foreground classification.

- The long term model captures a more stable representation of the scene background and adapts to changes slowly. This model consists of $\mathrm{N}$ sample pixels taken from a much larger window in time. The sample is updated using a non selective maintenance mechanism.

So, to combine the advantage of each model and to eliminate their disadvantages, the next foreground detection is obtained by taking the intersection of the two foreground detection coming from the short term model and the long term model. This intersection eliminates the persistence false positives detection from the short term model and extra false positives detection that occur in the long term model results. The only false positives detection that will remain will be rare events not represented in either model. If this rare event persists over time in the scene then the long term model will adapt to it, and it will be suppressed from the result later. Taking the intersection will, unfortunately, suppress true positives in the first model result that are false negatives in the second, because the long term model adapts to foreground as well if they are stationary or moving slowly. To address this problem, all pixels detected by the short term model that are adjacent to pixels detected by the combination are included in the final foreground detection.

Improvements: The original KDE present several advantages. The multimodality of the model allows dealing with multimodal backgrounds particularly in fast changes (waving trees, water rippling, etc...). Despite it, this model 
present some disadvantages: $\mathrm{N}$ frames need to be kept in memory during the entire detection process which is costly memory wise when $\mathrm{N}$ is large. The algorithm is time consuming too due the complexity in $\mathrm{O}\left(\mathrm{N}^{*} \mathrm{~N}\right)$. To solve these problems, different improvements have been proposed:

- Intrinsic improvements: These strategies consist in changing the kernel function [141-149] as shown in Table 8. For the training, some authors propose to decrease the number of samples by determining a proper size of the frame buffer [143], by using a diversity sampling scheme $[150,151]$ or by using a sequential Monte Carlo sampling scheme [152]. A recent patent concern the sequential kernel density approximation through mode propagation [359]. Furthermore, recursive maintenance [143-145,153, 154, 159] can be adopted to reduce the computation time. For the foreground detection, different scheme can be used as in [143, 146, 147, 153-155]. For the feature type, several features are used instead of the RGB space like the edge features [156] and motion features [157]. To choose which features to use, Parag et al. [158] proposed a framework for feature selection.
- Extrinsic improvements: Some authors (Table 9) used Markov Random Fields [155, 159], hierarchical approaches [160], multiple backgrounds [161] and graph cuts [162].

- Enhancing the foreground detection: Another way to improve this method is to enhance the results of the foreground detection by using cooperation with another segmentation method. It achieved by cooperation with the consecutive frame difference [163] or using a subspace learning approach using PCA [164].

The Table $\mathbf{8}$ and $\mathbf{9}$ give respectively an overview of the intrinsic and extrinsic improvements. Table $\mathbf{1 0}$ and Table $\mathbf{1 1}$ show respectively an overview of the critical situations and the real-time constraints for the different KDE versions that can tackle them better than the original one.

Table 8. Intrinsic improvements of the KDE

\begin{tabular}{|c|c|c|}
\hline Background Step & Improvements & Authors - References \\
\hline \multirow[t]{5}{*}{ Background Model } & Gaussian Kernel Function & $\begin{array}{l}\text { Automatic selection of kernel band width: Tavakkoli et al. } \\
{[141,142]}\end{array}$ \\
\hline & Rectangular Kernel Function & $\begin{array}{l}\text { Constant kernel band width: Ianasi et al. [143], Tanaka et al. } \\
{[144,145]} \\
\text { Variable kernel band width: Zivkovic [146] }\end{array}$ \\
\hline & Derivative Kernel Function & Cvetkovic et al. [147] \\
\hline & $\begin{array}{l}\text { Negative coefficient } \\
\text { polynomial kernel function }\end{array}$ & Witherspoon and Zhang [148] \\
\hline & Cauchy Kernel Function & Ramezani et al. [149] \\
\hline Background Initialization & $\begin{array}{l}\text { Decreasing the number of } \\
\text { samples }\end{array}$ & $\begin{array}{l}\text { Adopting the proper size of frame buffer: Ianasi et al. [143] } \\
\text { Diversity samples scheme: Mao and Shi }[150,151] \\
\text { Sequential Monte Carlo sampling: Tang et al. }[152]\end{array}$ \\
\hline \multirow{4}{*}{ Background Maintenance } & Background image & Ianasi et al. [143] \\
\hline & Recursive Maintenance & $\begin{array}{l}\text { Recursive maintenance of the PDF: Tavakkoli et al. [153], } \\
\text { Tanaka et al. [144, 145], Ramezani et al. [149] } \\
\text { Recursive maintenance of the background PDF and } \\
\text { foreground PDF: Tavakkoli et al. }[154] \\
\text { Recursive maintenance of the PDF and the background } \\
\text { image: Ianasi et al. [143] }\end{array}$ \\
\hline & Number of samples & Zivkovic [146] \\
\hline & Selective Maintenance & Tavakkoli et al. [141, 142], Mao and Shi [151] \\
\hline \multirow[t]{4}{*}{ Foreground Detection } & Dissimilarity measure & Ianasi et al. [143] \\
\hline & Probability & Zivkovic [146], Tavakkoli et al. [153] \\
\hline & Foreground model & Tavakkoli et al. $[153,154]$ \\
\hline & Two thresholds & Cvetkovic et al. [147] \\
\hline
\end{tabular}

Table 9. Extrinsic improvements of the KDE

\begin{tabular}{|l|l|}
\hline Methods & Authors - References \\
\hline \hline Markov Random Fields & Pahalawatta et al. [159] \\
\hline Hierarchical approaches & Orten et al. $[160]$ \\
\hline Multiples backgrounds & Tanaka et al. [161] \\
\hline Graph cuts & Mahamud [162] \\
\hline
\end{tabular}


Table 10. Challenges and KDE Versions

\begin{tabular}{|l|l|}
\hline Critical Situations & Authors - References \\
\hline \hline CS 1 - Noise Image & Mao and Shi [150, 151] \\
\hline CS 2-1 - Camera jitter & Sheihk and Shah [155] \\
\hline CS 2- 2 - Camera Adjustements & Cvetkovic et al. [147], Sung et al. [347], Hwang et al. [348] \\
\hline CS 3 - Gradual Illumination Changes & Sheihk and Shah [155] \\
\hline CS 4 - Sudden Illumination Changes & Sung et al. [48], Hwang et al. [49] \\
\hline CS 5-1 - Bootstrapping during initialization & Martel-Brisson and Zaccarin [346] \\
\hline CS 5-2 - Bootstrapping during maintenance & Sheihk and Shah [155] \\
\hline CS 6 - Camouflage & Tavakkoli et al. [142], Gu et al. [345] \\
\hline CS7 - Foreground Aperture & \\
\hline CS 8 - Moved background objects & Elgammal et al. [15], Cvetkovic et al. [147] \\
\hline CS 9 - Inserted background objects & \\
\hline CS 10 - Multimodal background & \\
\hline CS 11 - Waking foreground object & \\
\hline CS 12 - Sleeping foreground objects & \\
\hline CS 13 - Shadows Detection & Elgammal et al. [15], Cvetkovic et al. [147], Mao and Shi [150, 151] \\
\hline
\end{tabular}

Table 11. Real Time Constraints and KDE Versions

\begin{tabular}{|l|l|}
\hline Real-Time Constraints & Authors - References \\
\hline \hline Computation Time & Elgammal [349], Sadeghi et al. [350] \\
\hline Memory Requirement & Elgammal [349], Sadeghi et al. [350] \\
\hline
\end{tabular}

Discussion: The KDE is more adapted for outdoor scene where dynamic backgrounds appear but less suited for illumination changes.

\subsection{Subspace Learning using PCA (SL-PCA)}

Subspace learning offer a good framework to deal with illumination changes as it allows taking into account spatial information. Oliver et al. [165] proposed to model each background pixel using an eigenbackground model.

This model consists in taking a sample of $\mathrm{N}$ images $\left\{I_{1}, I_{2}, \ldots, I_{N}\right\}$ and computing the mean background image $\mu_{B}$ and its covariance matrix $C_{B}$.

This covariance matrix is then diagonalized using an eigenvalue decomposition as follows:

$$
L_{B}=\Phi_{B} C_{B} \Phi_{B}^{T}
$$

where $\Phi_{B}$ is the eigenvector matrix of the covariance of the data and $L_{B}$ is the corresponding diagonal matrix of its eigenvalues.

In order to reduce, the dimensionality of the space, only $\mathrm{M}$ eigenvectors $(\mathrm{M}<\mathrm{N})$ are kept in a principal component analysis (PCA). The M largest eigenvalues are contained in the matrix $L_{M}$ and the $\mathrm{M}$ vectors correspond to these $\mathrm{M}$ largest eigenvalues in the matrix $\Phi_{M}$.
Once the eigenbackground images stored in the matrix $\Phi_{M}$ are obtained and the mean $\mu_{B}$ too, the input image $I_{t}$ can be approximated by the mean background and weighted sum of the eigenbackgrounds. $\Phi_{M}$.

The coordinate in eigenbackground space of input image $I_{t}$ can be computed as follows:

$$
w_{t}=\left(I_{t}-\mu_{B}\right)^{T} \Phi_{M}
$$

When $w$ is back projected onto the image space, a reconstructed background image is created as follows:

$$
B_{t}=\Phi_{M} w_{t}^{T}+\mu_{B}
$$

Then, the foreground object detection is made as follows:

$$
\left|I_{t}-B_{t}\right|>T
$$

where $\mathrm{T}$ is a constant threshold.

Improvements: The eigenbackground model which we have called SL-PCA provides a robust model of the probability distribution function of the background, but not of the moving objects while they do not have a significant contribution to the model. So, the first limitation of this model is that the size of the foreground object must be small and don't appear in the same location during a long period in the training sequence. The second limitation appears for the background maintenance. Indeed, it is computationally intensive to perform model updating using the batch mode 
PCA. Moreover without a mechanism of robust analysis, the outliers or foreground objects may be absorbed into the background model. The third limitation is that the application of this model is mostly limited to the gray-scale images since the integration of multi-channel data is not straightforward. It involves much higher dimensional space and causes additional difficulty to manage data in general. Another limitation is that the representation is not multimodal so various illumination changes cannot be handled correctly. To alleviate these limitations, numerous improvements (25 papers) have been proposed over the recent years. A survey over 15 papers in the period 19992009 can be found in [334]. Thus, the different improvements which attempt to solve these four limitations are summarized in the following classification with the recent advances:

Alleviate the limitation of the size of the foreground object: $\mathrm{Xu}$ et al. [166, 167] proposed to apply recursively an error compensation process which reduces the influence of foreground moving objects on the eigenbackground model. An adaptive threshold method is also introduced for background subtraction, where the threshold is determined by combining a fixed global threshold and a variable local threshold. Results show more robustness in presence of moving objects. Another approach developed by Kawabata et al. [168] consists in an iterative optimal projection method to estimate a varied background in real time from a dynamic scene with foreground. Firstly, background images are collected for a while and then the background images are compressed using eigenspace method to form a database. After this initialization, a new image is taken and projected onto the eigenspace to estimate the background. As the estimated image is much affected by the foreground, the foreground region is calculated by using background subtraction with former estimated background to exclude the region from the projection. Thus the image whose foreground region is replaced by the former background is projected to eigenspace and then the background is updated. Kawabata et al. [25] proved that the cycle converges to a correct background image. Recently, Quivy and Kumazawa [351] proposed to generate the background images using the Nelder-Mead Simplex algorithm and a dynamic masking procedure. This paper presents an original method that replaces the projection/reconstruction step of the SL-PCA by a direct background image generation. The experiments proved that the proposed method performs better then than the SL-PCA [165], SL-REC [166, 167], and SL-IOP [168] for large and fast moving objects.

Dealing with the time requirement and the robustness: For the maintenance, some authors [169-177] proposed different algorithms of incremental PCA. The incremental PCA proposed by [169] need less computation but the background image is contamined by the foreground object. To solve this, Li et al. [170, 171] proposed an incremental PCA which is robust in presence of outliers. However, when keeping the background model updated incrementally, it assigned the same weights to the different frames. Thus, clean frames and frames which contain foreground objects have the same contribution. The consequence is a relative pollution of the background model. In this context, Skocaj et al. [172, 173] used a weighted incremental and robust. The weights are different following the frame and this method achieved a better background model. However, the weights were applied to the whole frame without considering the contribution of different image parts to building the background model. To achieve a pixel-wise precision for the weights, Zhang and Zhuang [174] proposed an adaptive weighted selection for an incremental PCA. This method performs a better model by assigning a weight to each pixel at each new frame during the update. Experiments [174] show that this method achieves better results than the SL-IRPCA [170, 171]. Wang et al. [175, 176] used a similar approach using the sequential Karhunen-Loeve algorithm. Recently, Zhang et al. [209] improved this approach with an adaptive scheme. All these incremental methods avoid the eigen-decomposition of the high dimensional covariance matrix using approximation of it and so a low decomposition is allowed at the maintenance step with less computational load. However, these incremental methods maintain the whole eigenstructure including both the eigenvalues and the exact matrix $\Phi_{M}$. To address this problem, Li et al. [177] proposed a fast recursive and robust eigenbackground maintenance avoiding eigendecomposition. This method achieves similar results than the SL-IPCA [169] and the SL-IRPCA [170, 171] at better frames rates. Fig. (4). shows a classification of these algorithms following their robustness and their adaptivity.

Dealing with the grey scale and the pixel-wise limitations: Recently, $\mathrm{Wu}$ et al. [207] proposed to combine the PCA model with single gaussian model. PCA allow the robustness to illumination changes and the single gaussian to describe color information for each pixel. So, it can detect the chroma changes and remove shadow pixels. An adaptively strategy is used to integrate the two models. A binary graph cut is then used to perform the foreground/background segmentation. In another way, Han and Jain [178] proposed an efficient algorithm using a weighted incremental 2-Dimensional Principal Component Analysis. It is shown that the principal components in 2DPCA are computed efficiently by transformation to standard PCA. To perform the computational time, Han and Jain [178] used an incremental algorithm to update eigenvectors to handle temporal variations of background. The proposed algorithm was applied to 3channel (RGB) and 4-channel (RGB+IR) data. 


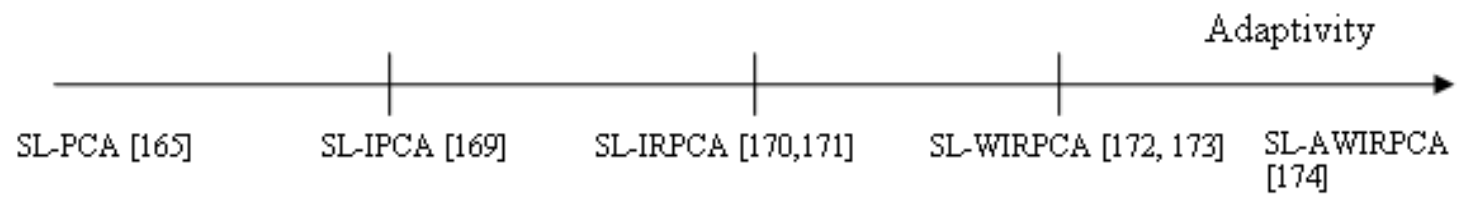

Fig. (4): Adaptivity of the SL-PCA Algorithms

Results show noticeable improvements in presence of multimodal background (MB) and shadows (S). To solve the pixel-wise limitation, Zhao et al. [206] used spatio-temporal block instead of pixel. Furthermore, their method consist in applying the candid covariance free incremental principal components analysis algorithm (CCIPCA) which is fast in convergence rate and low in computational complexity than classical IPCA algorithms. Results show more robustness robust to noise and fast lighting changes.

- Dealing with multimodal illumination changes: Recently, Dong et al. [211] proposed to use a multi-subspace learning to handle different illumination changes. The feature space is organized into clusters which represent the different lighting conditions. A Local Principle Component Analysis (LPCA) transformation is used to learn separately an eigen-subspace for each cluster. When a current image arrives, the algorithm selects the learned subspace which shares the nearest lighting condition. The results [211] show that the LPCA algorithm outperforms the original PCA [165] algorithm and MOG [14] especially under sudden illumination changes. In a similar way, Kawanishi et al. [213-214] generated the background image which well expresses the weather and the lighting condition of the scene. This method collects a huge number of images by super long term surveillance, classifies them according to their time in the day, and applies the PCA so as to reconstruct the background image.

A recent patent concern a method based on space-time video block and online subspace learning [360]. This method allows a robust incremental update and alleviates the pixelwise limitations.

The Table 12, Table 13, Table 14 and Table 15 group by type the different improvements of the SL-PCA.

Table 12. Influence of the foreground objects

\begin{tabular}{|l|l|}
\hline Methods & Authors - Dates \\
\hline \hline $\begin{array}{l}\text { Recursive Error } \\
\text { Compensation } \\
\text { (SL-REC) }\end{array}$ & Xu et al. $(2006)[166,167]$ \\
\hline $\begin{array}{l}\text { Iterative Optimal } \\
\text { Projection } \\
\text { (SL-IOP) }\end{array}$ & Kawabata et al. $(2006)[168]$ \\
\hline $\begin{array}{l}\text { Simplex Algorithm } \\
\text { (SL-SA) }\end{array}$ & Quivy and Kumazawa (2011) [351] \\
\hline
\end{tabular}

Table 13. Time requirement and the robustness

\begin{tabular}{|l|l|}
\hline Methods & Authors - Dates \\
\hline \hline $\begin{array}{l}\text { Incremental PCA } \\
\text { (SL-IPCA) }\end{array}$ & Rymel et al. (2004) \\
\hline $\begin{array}{l}\text { Incremental and robust PCA } \\
\text { (SL-IRPCA) }\end{array}$ & Li et al. (2003) [170, \\
\hline Weighted Incremental and Robust & Skocaj et al. (2003) \\
PCA (SL-WIRPCA) & {$[172,173]$} \\
\hline $\begin{array}{l}\text { Adaptive Weight Selection for } \\
\text { Incremental PCA (SL-AWIPCA) }\end{array}$ & Zhang and Zhuang \\
\hline $\begin{array}{l}\text { Sequential Karhunen-Loeve } \\
\text { algorithm (SL-SKL) }\end{array}$ & Wang et al. (2006) \\
\hline $\begin{array}{l}\text { Adaptive Sequential Karhunen- } \\
\text { Loeve algorithm (SL-ASKL) }\end{array}$ & {$[175,176]$} \\
\hline $\begin{array}{l}\text { Fast Recursive Maintenance } \\
\text { (SL-FRM) }\end{array}$ & Zhang et al. [209] \\
\hline
\end{tabular}

Table 14. Dealing with the grey scale and the pixel-wise limitations

\begin{tabular}{|l|l|}
\hline Methods & Authors - Dates \\
\hline \hline PCA - Single Gaussian & Wu et al. (2009) [207, 208] \\
(SL-PCA-SG) & \\
\hline $\begin{array}{l}\text { Weighted Incremental 2PCA } \\
\text { (SL-WI2DPCA) }\end{array}$ & Han and Jain (2007) [178] \\
\hline $\begin{array}{l}\text { Candid Covariance } \\
\text { Incremental PCA } \\
\text { (SL-CCIPCA) }\end{array}$ & Zhao et al. (2008) [206] \\
\hline
\end{tabular}

Table 15. Dealing with multimodal illumination changes

\begin{tabular}{|l|l|}
\hline Methods & Authors - Dates \\
\hline \hline $\begin{array}{l}\text { Local Principle Component } \\
\text { Analysis on Clusters } \\
\text { (LPCA-C) }\end{array}$ & $\begin{array}{l}\text { Dong et al. } \text { (2010) [211, } \\
212]\end{array}$ \\
\hline $\begin{array}{l}\text { Local Principle Component } \\
\text { Analysis on Separated }\end{array}$ & Kawanishi et al. (2009) \\
$\begin{array}{l}\text { Sequences } \\
\text { (LPCA-SS) }\end{array}$ & {$[213-214]$} \\
\hline
\end{tabular}

\subsection{Discussion}

In Section 3, we surveyed the models of the first category and their related improvements. These improvements perform each original algorithm for specified critical situations. However, some authors have recently proposed to use more advanced statistical models as Support Vector models to deal more accurately with dynamics background. 


\section{SECOND CATEGORY}

The second category models use more sophisticated statistical model as support vector machine (SVM), support vector regression (SVR) and support vector data description (SVDD).

\subsection{Support Vector Machine (SVM)}

Support Vector Machines were introduced by Vapnik et al. [179]. For classification, SVMs work by determining a hyperplane in a high dimensional feature space to separate the training data into two classes. The best hyperplane can be derived by minimizing the margin which represents the least distance from the hyperplane to the data. Using this classification aspect, Lin et al. [180] proposed to use the SVMs for background modeling. Particularly, Lin et al. [180] used a PSVM with probabilistic outputs because the SVM gives only binary ouputs. A sigmoid model is used to convert binary SVM scores into posterior probabilities:

$$
p(y=1 \mid f)=\frac{1}{1+\exp (A f+B)}
$$

where $\mathrm{y}$ is binary class label and $\mathrm{f}$ is an output score of the SVM decision function. The two parameters $\mathrm{A}$ and $\mathrm{B}$ are fitted using maximum likelihood estimation from a training set $\left(f_{i}, y_{i}\right)$, and derived by minimizing the negative loglikelihood function:

$$
\min -\sum_{t} t_{i} \log \left(p_{i}\right)+\left(1-t_{i}\right) \log \left(1-p_{i}\right)
$$

where

$$
t_{i}=\frac{y_{i}+1}{2} \text { and } p_{i}=\frac{1}{1+\exp \left(A f_{i}+B\right)}
$$

To avoid overfitting and to derive unbiased training for the minimization, a hold-out set is generated from the data by dividing each training set of $80 \%$ and $20 \%$ respectively. The large subset is used for SVM training, and the smaller one is used for the two parameter minimization. In this context, Lin et al. [180] used 100 images of size $160 * 120$ with known background. Each image is divided into blocks of size $4 * 4$ and considering two features for each block: optical flow value and consecutive image difference. For each block, its label is defined as +1 for background and -1 otherwise. The background initialization starts with the first image and each block are tested by the PSVM. An image block is classified as background if its probability output is larger than a threshold T:

$$
p\left(b_{i}\right)>T
$$

When an image block $p\left(b_{i}\right)>T$ is classified as background for $\mathrm{M}$ consecutive times, the Fisher linear distance is used:

$$
d\left(b_{i}, b_{\text {back }}\right)=\frac{\left(\mu_{i}-\mu_{\text {back }}\right)^{2}}{\left(\sigma_{i}^{2}-\sigma_{\text {back }}^{2}\right)}(\mathbf{2 6})
$$

where $\mu$ and $\sigma^{2}$ are the mean and the variance of the intensity distribution of a block.

When the distance between the two blocks is large, two possible conditions appear. The current block can be either part of a uniform region of a moving object or a new background just revealed. The averaging PSVM probability for the current block over the past $\mathrm{M}$ frames is compared with the PSVM probability of the background. If the new average PSVM probability is larger, then the background is replaced by the current block.

Continuing this way, the initialization process will be terminated when replacement events do not occur for a consecutive $\mathrm{M}$ frames. When the initialization is finished, the foreground detection is made by thresholding the difference between the background model and the current image.

\subsection{Support Vector Regression (SVR)}

Given a set of training data, SVR fits a function by specifying an upper bound on a fraction of training data allowed to lie outside of a distance $\varepsilon$ from the regression estimate. This type of SVR is usually referred to as $\varepsilon$ insensitive SVR [181]. For each pixel belonging to the background, a separate SVR is used to model it as a function of intensity. To classify a given pixel as background or not, Wang et al. [183] [184] feed its intensity value to the SVR associated it and threshold the output of the SVR. Let assume a set of training data for some pixel $\mathrm{p}$ obtained from several frames $\left\{\left(\mathrm{x}_{1}, \mathrm{y}_{1}\right), \ldots,\left(\mathrm{x}_{\mathrm{N}}, \mathrm{y}_{\mathrm{N}}\right)\right\}$, where $\mathrm{x}_{\mathrm{i}}$ corresponds to the intensity value of pixel $p$ at frame $i$, and $y_{i}$ corresponds to the confidence of pixel $p$ being a background pixel. Once the SVR has been trained, the confidence of the pixel $p$ in a new frame $\mathrm{i}, \mathrm{f}\left(\mathrm{x}_{\mathrm{i}}\right)$, is computed using the following linear regression function:

$$
f\left(x_{i}\right)=\sum_{j=1}^{N}\left(a_{i}-a_{j}^{*}\right) k\left(x_{i}, x_{j}\right)+\xi
$$

where $k\left(x_{i}, x_{j}\right)$ is a kernel function. The parameters $a$, $a^{*}$ and $\xi$, called Lagrange multipliers, are obtained by solving an optimization problem using the method of the Lagrange multipliers. Given the SVR-based background model, the intensity of each pixel in a new frame forms the input to the SVR. The output of the SVR represents the confidence that a given pixel belongs to the background. Eventually, a pixel is labelled as background if its 
confidence is between a low threshold $S_{1}$ and a high threshold $S_{h}$. Specifically, a binary foreground detection map is formed at frame $t$ as follows:

$$
\begin{aligned}
& M_{x_{i}}^{t}=0 \text { if } S_{l}<f\left(x_{i}\right)<S_{b} \\
& M_{x_{i}}^{t}=1 \text { otherwise }
\end{aligned}
$$

where $f\left(x_{i}\right)$ is the SVR output and $S=\left\{S_{l}, S_{h}\right\}$ are the initial thresholds. Then, for each region in the binary map, the SVR-based background model is update using an online SVR learning algorithm [182].

\subsection{Support Vector Data Description (SVDD)}

Tavakkoli et al. [186] proposed to model the background using support vector data description (SVDD) in videos with quasi-stationary backgrounds. Data domain description concerns the characteristics of a data set [185]. The boundary of the dataset can be used to detect novel data or outliers. A normal data description gives a closed boundary around the data. The simplest boundary can be represented by a hypersphere. The volume of this hyper-sphere with center $a$ and radius $R$ should be minimized while containing all the training samples $\chi_{i}$. To allow the possibility of outliers in the training set, slack variables $\mathcal{E}_{i} \geq 0$ are introduced. The error function to be minimized is defined as:

$$
F(R, a)=R^{2}+C \sum_{i} \varepsilon_{i}
$$

Subjects to the contraints:

$$
\left\|x_{i}-a\right\|^{2} \leq \mathrm{R}^{2}+\varepsilon_{i} \forall i
$$

In equation (1), $\mathrm{C}$ is a trade-off between simplicity of the system and its error and is called confidence parameter. After incorporating the constraints (30) into the error function (29) by Lagrange multipliers we have:

$$
L\left(\mathrm{R}, a, \alpha_{i}, \gamma_{i}, \varepsilon_{i}\right)=\mathrm{R}^{2}+C \sum_{i} \varepsilon_{i}-\sum \alpha_{i}\left(\mathrm{R}^{2}+\varepsilon_{i}-\left(\left\|x_{i}-a\right\|^{2}\right)\right)-\sum_{i} \gamma_{i} \varepsilon_{i}
$$

L should be maximized with respect to Lagrange multipliers $\alpha_{i} \geq 0$ and $\gamma_{i} \geq 0$ and minimized with respect to $\mathrm{R}, a$ and $\varepsilon_{i}$. Lagrange multipliers $\gamma_{i}$ can be removed if the constraint $0 \leq \alpha_{i} \leq C$ is imposed. After solving the optimization problem we have:

$$
\begin{aligned}
& L=\sum_{i} \alpha_{i}\left(x_{i} \cdot x_{i}\right)-\sum_{i, j} \alpha_{i} \alpha_{j}\left(x_{i} \cdot x_{j}\right) \\
& \forall \alpha_{i}: 0 \leq \alpha_{i} \leq C
\end{aligned}
$$

When a new sample satisfies the inequality in (30), then its corresponding Lagrange multipliers are $\alpha_{i} \geq 0$, otherwise they are zero.

Therefore we have:

$$
\begin{aligned}
& \left\|x_{i}-a\right\|^{2}<\mathrm{R}^{2} \rightarrow \alpha_{i}=0, \gamma_{i}=0 \\
& \left\|x_{i}-a\right\|^{2}>\mathrm{R}^{2} \rightarrow \alpha_{i}=C, \gamma_{i}>0
\end{aligned}
$$

From the above, we can remark that only samples with nonzero $\alpha_{i}$ are needed in the description of the data set, therefore they are called support vectors of the description. To test a new sample $y$, its distance to the center of the hyper-sphere is calculated and tested against R. Tavakkoli et al. [186] used this methodology to built a descriptive boundary for each pixel in the background training frames to generate its model for the background. Then, these boundaries are used to classify their corresponding pixels in new frames as background and foreground pixels. In practice, for each pixel in the scene a single class classifier is trained by using its values in the background training frames. This classifier consists of the description boundary and support vectors, as well as a threshold used to describe the data. For the foreground detection, each pixel in the new frames is classified as background or foreground using its value and its corresponding classifier from the training stage. Feature vectors $\mathrm{x}_{\mathrm{ij}}$ used in the current implementation are $\mathrm{x}_{\mathrm{ij}}=\left[\mathrm{C}_{\mathrm{r}}\right.$; $\mathrm{C}_{\mathrm{g}}$ ], where $\mathrm{C}_{\mathrm{r}}$ and $\mathrm{C}_{\mathrm{g}}$ are the red and green chrominance values for pixel $(\mathrm{i}, \mathrm{j})$.

Improvements: This model presents several advantages: The accuracy is not bounded to the accuracy of the estimated probability density functions and the memory requirement is less than non-parametric techniques. Because support vector data description explicitly models the decision boundary of the known class, it is suitable for novelty detection without the need to use thresholds. Furthermore, the classifier performance in terms of false positive is controlled explicitly. The main disadvantage is that the training of SVDD requires a Lagrange optimization which is computationally intensive. For the maintenance, all the SVDD must be recomputed. To perform the training, Tavakkoli et al. [187] proposed to use a genetic approach to solve the Lagrange optimization problem. The Genetic Algorithm (GA) starts with the initial guess and solves the optimization problem iteratively. In [188][189], Tavakkoli et al. proposed to use an incremental SVDD. In this way, the maintenance is performed too.

\subsection{Discussion}

Support vector models offer a nice framework for background modeling specifically in presence of illumination changes and dynamic backgrounds. Another way to model the background is to perform the first category by using a more adaptive model. 


\section{THIRD CATEGORY}

The third category models generalize the first category model as the single general Gaussian (SGG), the mixture of general Gaussians (MOGG) and subspace learning using Incremental Component Analysis (SL-ICA), Incremental Non-negative Matrix Factorization (SL-INMF) or Incremental Rank- $\left(\mathrm{R}_{1}, \mathrm{R}_{2}, \mathrm{R}_{3}\right)$ Tensor (SL-IRT).

\subsection{Single General Gaussian (SGG)}

Kim et al. [190-192] proposed to model the background using a generalised Gaussian family (GGF) model of distributions to cope with problems from various changes in background and shadows. The idea is that pixel variance fitted sometimes a Laplace one or a Gaussian one. Indeed, pixel variance in a static scene over time in indoor scenes taken with the latest camera is closer to a Laplace distribution than a Gaussian, but the Laplace model has limitation for use in various environments. The pixel variation in a static scene over time is defined as:

$$
\begin{gathered}
P\left(X_{t}\right)=\frac{\rho \gamma}{2 \Gamma(1 / \rho)} e^{-\left(\gamma^{p}|x-\mu|^{p}\right)} \text { with } \\
\gamma=\frac{1}{\sigma}\left(\frac{\Gamma(3 / \rho)}{\Gamma(1 / \rho)}\right)
\end{gathered}
$$

where $\Gamma(\bullet)$ is a gamma function and $\sigma^{2}$ is a variance of the distribution. In Equation (1), $\rho=1$ represents a Laplace distribution while $\rho=2$ represents a Gaussian distribution. The models are decided for each pixel by computing excess kurtosis $g_{2}$ of the first $\mathrm{m}$ frames. The excess kurtosis of Laplace and Gaussian distributions is respectively 3 and 0. The optimal parameters of the background model are estimated by the maximization of the likelihood of the observed value:

$$
g_{2}=\frac{N \sum_{i=1}^{N}\left(x_{i}-\mu\right)^{4}}{\left(\sum_{i=1}^{N}\left(x_{i}=\mu\right)^{2}\right)^{2}}-3
$$

In practice, Kim et al. [190-192] modelled the background in two parts: a luminance component obtained by a weighted mean of RGB channels and a hue component in HSI color space. The maintenance is made using a selective running average as in [13]. The foreground detection is firstly performed by subtracting the intensity components of the current frame from the background model:

$$
D(x, y)=|I(x, y)-B(x, y)|
$$

where $I(x, y)$ and $B(x, y)$ correspond respectively to the luminance of the current frame and the background model. Then, pixels are classified into three categories using two thresholds as follows:

$$
\begin{aligned}
& \text { background pixel if } D(x, y)<T_{1} k(x, y) \\
& \text { suspicious pixel if } T_{1} k(x, y) \leq D(x, y) \leq T_{2} k(x, y) \\
& \text { foreground pixel if } T_{2} k(x, y)<D(x, y)
\end{aligned}
$$

where $k(x, y)$ is a scale parameter. The thresholds $T_{1}, T_{2}$ and $T_{3}$ are determined using the training frames. The SGG shows better performance than the MOG and the KDE in indoor and outdoor scene.

\subsection{Mixture of General Gaussians (MOGG)}

Allili et al. [193-195] proposed a finite mixture model of general Gaussians for robust segmentation in the presence of noise and outliers. This model has more flexibility to adapt the shape of data and less sensibility for over-fitting the number of classes than the mixture of Gaussians. Each pixel is characterized by its intensity in the RGB color space. Then, the probability of observing the current pixel value is considered given by the following formula in the multidimensional case:

$$
P\left(X_{t}\right)=\sum_{i=1}^{K} \omega_{i, t} . \eta\left(X_{t}, \mu_{i, t}, \sigma_{i, t}, \lambda_{i}\right)
$$

where the parameters are $\mathrm{K}$ is the number of distributions, $\omega_{i, t}$ is a weight associated to the $\mathrm{i}^{\text {th }}$ Gaussian at time $\mathrm{t}$ with mean $\mu_{i, t}$ and standard deviation $\sum_{i, t} \cdot \lambda_{i}=0$ if the distribution is a Gaussian one and $\lambda_{i}=3$ if the distribution is a Laplace one. $\eta$ is a Gaussian probability density function:

$$
\begin{aligned}
& \eta\left(X_{t}, \mu_{i, t}, \sigma_{i, t}, \lambda_{i}\right)=\prod_{j=1}^{d} A\left(\lambda_{j}\right) \exp \left(-B\left(\lambda_{i}\right)\left|\frac{X_{j}-\mu_{j}}{\sigma_{j}}\right|^{\lambda_{j}}\right) \\
& \text { where } A(\lambda)=\frac{(\Gamma(3 / \lambda) / \Gamma(1 / \lambda))^{1 / \lambda}}{2 \sigma \Gamma(1 / \lambda)} \text { and } \\
& B(\lambda)=\left(\frac{\Gamma(3 / \lambda)}{\Gamma(1 / \lambda)}\right) \text {. }
\end{aligned}
$$

The optimal number of Gaussians is computed at each time $t$ by minimizing the criterion Minimum Message Length (MML). If the number of Gaussians at time $t+1$ is smaller than at time $t$, the parameters are updated in a similar way than in [14]. The same matching test as in [14] is used to check if a pixel matches a Gaussian. For the labeling, the same scheme that Stauffer and Grimson [14] is used. The MOGG show better performance than the MOG in the presence of shadows $(\mathrm{S})$. 


\subsection{Subspace Learning}

Subspace learning can be made using PCA as seen in the Section 3.4. In the literature [196], there are other methods to reduce the space and these different methods have been classified by Skocaj and Leonardis [197] as reconstructive methods and discriminative methods:

Reconstructive subspace learning: The reconstructive methods allow a well approximation of data and so provide a good reconstruction. Another advantage is that reconstructive methods are unsupervised techniques. Furthermore, reconstructive methods enable incremental updating which is very suitable for real-time application. These methods are task-independents. The most common reconstructive methods are the following: Principal Components Analysis (PCA) [51], Independent Component Analysis (ICA) [52] and Nonnegative Matrix Factorization (NMF) [53]. PCA transforms a number of possibly correlated data into a smaller number of uncorrelated data called principal components. ICA is a variant of PCA in which the components are assumed to be mutually statistically independent instead of merely uncorrelated. The stronger condition allows remove the rotational invariance of PCA, i.e. ICA provides a meaningful unique bilinear decomposition of two-way data that can be considered as a linear mixture of a number of independent source signals. Non-negative matrix factorization (NMF) finds linear representations of nonnegative data. Given a non-negative data matrix $\mathrm{V}$, $\mathrm{NMF}$ finds an approximate factorization $\mathrm{V}=\mathrm{WH}$ into non-negative factors $\mathrm{W}$ and $\mathrm{H}$. The non-negativity constraints make the representation purely additive, i.e allowing no subtractions, in contrast to principal component analysis (PCA) and independent component analysis (ICA).

Discriminative subspace learning: The discriminative methods are supervised techniques and allow a well separation of data and so provide a good classification. Furthermore, discriminative methods are spatially and computationally efficient. These methods are taskdependents. The most common discriminative methods are the following: Linear Discriminant Analysis (LDA) [54] and Canonical Correlation Analysis (CCA) [55]. LDA projects the data onto a lower-dimensional vector space such that the ratio of the between-class distance to the within-class distance is maximized. The goal is to achieve maximum discrimination. Canonical correlation analysis (CCA) is a multivariate statistical model that facilitates the study of interrelationships among sets of multiple dependent variables and multiple independent variables. Canonical correlation simultaneously predicts multiple dependent variables from multiple independent variables.

All these methods are originally implemented with batch algorithms which require that the data must be available in advance and be given once altogether. However, this type of batch algorithms is not adapted for the application of background modeling in which the data are incrementally received from the camera. Furthermore, when the dimension of the dataset is high, both the computation and storage complexity grow dramatically. Thus, incremental methods are highly needed to compute in real-time the adaptive subspace for the data arriving sequentially. Following these constraints, the reconstructive methods are the most adapted for background modeling. Furthermore, their unsupervised aspect allows avoid a manual intervention in the learning step. In the following paragraphs, we survey the subspace leaning methods applied recently to background modeling: Independent Component Analysis (ICA), Non-negative Matrix Factorization (NMF) and Incremental Rank- $\left(\mathrm{R}_{1}, \mathrm{R}_{2}, \mathrm{R}_{3}\right)$ Tensor.

\subsubsection{Subspace learning using ICA (SL-ICA)}

ICA generalizes the technique of PCA. When some mixtures of probabilistically independent source signals are observed, ICA recovers the original source signals from the observed mixtures without knowing how the sources are mixed. The assumption made is that the observation vectors $X=\left(x_{1}, x_{2}, \ldots, x_{M}\right)^{T}$ can be represented in terms of a linear superposition of unknown independent vectors $S=\left(s_{1}, s_{2}, \ldots, s_{M}\right)^{T}$ :

$$
X=A S(\mathbf{4 0 )}
$$

where $A$ is an unknown mixing matrix $(\mathrm{M} \times \mathrm{N})$. ICA finds a matrix $\mathrm{W}$, so that the resulting vectors:

$$
Y=W X
$$

recovers the independent vectors $S$, probabilistically permuted and rescaled. $\mathrm{W}$ is roughly the inverse matrix of $\mathrm{A}$. Applying it to background modeling, the ICA model is given by:

$$
Y=W X_{t}(\mathbf{4 2})
$$

$X_{t}=\left(x_{B}, x_{F}\right)^{T}$ is the mixture data matrix of size $2 * \mathrm{~K}$ in which $\mathrm{K}=\mathrm{M} * \mathrm{~N} . x_{1}=\left(x_{11}, x_{12}, \ldots, x_{1 K}\right)$ is the first frame which can contain or not foreground objects and $x_{2}=\left(x_{i 1}, x_{22}, \ldots, x_{2 K}\right)$ is the second frame which contain foreground objects. $W=\left(w_{1}, w_{2}\right)^{T}$ is the demixing matrix, in which $w_{i}=\left(w_{i 1}, w_{i 2}\right)$ with $\mathrm{i}=1,2$. $Y=\left(y_{1}, y_{2}\right)^{T}$ is the estimated source signals in which $y_{i}=\left(y_{i 1}, y_{i 2}, \ldots, y_{i k}\right)$. Several ICA algorithms can be used to determine W. Yamazaki et al. [198] used a neural learning algorithm [199]. In another way, Tsai and Lai [200] used a Particle Swarm Algorithm (PSO) [201]. Once W is 
determined, there are two ways in the literature to generate the background and the foreground mask images:

- The first case which $x_{1}$ contains foreground object like in Yamazaki et al. [198]. Then, the foreground mask for the frames $x_{1}$ and $x_{2}$ is obtained by thresholding respectively $y_{1}$ and $y_{2}$. The background image is obtained by replacing regions representing foreground objects in $x_{1}$ by the corresponding regions representing background in $x_{2}$.

- The second case which $x_{1}$ contains no foreground object like in Tsai and Lai [200]. Then, the foreground mask for the frames $x_{2}$ is obtained by thresholding $y_{2}$. The background image is $y_{1}$.

The ICA model was tested on traffic scenes by Yamazaki $e t$ al. [198] and show robustness in changing background like illumination changes. In [200], the algorithm was tested on indoor scenes where sudden illumination changes appear.

\subsubsection{Subspace learning using INMF (SL-INMF)}

The non-negative matrix factorization (NMF), with rank $r$, decomposes the data matrix $V \in \mathrm{R}^{p \times q}$ into two matrices which are $W \in \mathrm{R}^{p \times r}$ called the mixing matrix, and $H \in \mathrm{R}^{r \times q}$ named as the encoding matrix:

$$
V \approx W H
$$

So, NMF aims to find an approximate factorization that minimizes the reconstruction error. Different cost functions based on the reconstruction error have been defined in the literature, but because of its simplicity and effectiveness, the squared error is the most used:

$$
F=\|V-W H\|^{2}=\sum_{i=1}^{p} \sum_{i=1}^{q}\left(V_{i j}-(W H)_{i j}\right)^{2}
$$

where subscription ij stands for the $\mathrm{ij}^{\text {th }}$ matrix entity. Applying it to background modelling, Bucak et al. [202, 203] proposed an incremental NMF algorithm. The background initialization is made using $\mathrm{N}$ training frames. So, $\mathrm{V}$ is vector column corresponding to a matrix of $\operatorname{size}(p \times q) \times N$. The matrices $\mathrm{W}$ and $\mathrm{H}$ are updated incrementally. The foreground detection is made by thresholding the residual error which correspond to the deviations between the background model and the projection of the current frame onto the background model. The INMF has similar performance to dynamic background and illumination changes than the IRPCA proposed by Li et al. [170].

\subsubsection{Subspace learning using Incremental Rank- (R1,R2, R3) Tensor (SL-IRT)}

The different previous subspace learning considered image as a vector. So, the local spatial information is almost lost. Li et al. [204, 205] proposed to use a high-order tensor learning algorithm called incremental rank- $\left(\mathrm{R}_{1}, \mathrm{R}_{2}, \mathrm{R}_{3}\right)$ tensor based subspace learning to take into account the spatial information. This online algorithm constructs a low-order tensor eigenspace model in which the sample mean and the eigenbasis are updated adaptively. Denote $G=\left\{B M_{q} \in R^{M \times N}\right\}_{q=1,2, \ldots, t}$ as a scene's background appearance sequence with the q-th frame being $B M_{q}$. Denote $p_{x y}$ as the $\mathrm{x}$-th and the $\mathrm{y}$-th pixel of the scene. The tensor-based eigenspace model for an existing $A=\left\{B M_{q}^{x y} \in R^{I_{1} \times I_{2} \times t}\right\}_{q=1,2, \ldots, t}\left(\mathrm{I}_{1}=\mathrm{I}_{2}=5\right.$ corresponding to a $\mathrm{K}$ neigborhood of $p_{w v}$ with $\mathrm{K}=\mathrm{I}_{1} \mathrm{I}_{2}-1=24$ ) consists of the maintained eigenspace dimensions $\left(\mathrm{R}_{1}, \mathrm{R}_{2}, \mathrm{R}_{3}\right)$ corresponding to the three tensor unfolding modes, the mode- $n$ column projection matrices $U^{(n)} \in \mathrm{R}^{I_{n} \times R_{n}}$, the mode-3 row projection matrix $V^{(3)} \in \mathrm{R}^{\left(I_{n} \cdot I_{2}\right) \times \mathrm{R}_{3}}$, the column means $\bar{L}^{(1)}$ and $\bar{L}^{(2)}$ of the mode-(1,2) unfolding matrices $A_{(1)}$ and $A_{(2)}$, and the row mean $\bar{L}^{(3)}$ of the mode-3 unfolding matrix $A_{(3)}$. Given the K-neighbor image region $I_{t+1}^{u v} \in \mathrm{R}^{I_{1} \times I_{2} \times 1}$ centered at the $\mathrm{x}$-th and $\mathrm{y}$-th pixel $p_{x y}$ of the current incoming frame $I_{t+1} \in \mathrm{R}^{M \times N \times 1}$, the distance $R M_{x y}$ (determined by the three reconstruction error norms of the three modes) between $I_{t+1}^{x y}$ and the learned tensorbased eigenspace model is computed. Then, the foreground detection is defined as follows:

$p_{x y}$ is classified as background if $\exp \left(-\frac{R M_{x y}^{2}}{2 \sigma^{2}}\right)>T$

$p_{x y}$ is classified as foreground otherwise

where $\sigma$ is a scaling factor and Tenotes a threshold. Thus, the new background model $B M_{t+1}(x, y)$ at time $t+1$ is defined as:

$B M_{t+1}(x, y)=H_{x y} \quad$ if $p_{x y}$ is classified as foreground

$B M_{t+1}(x, y)=I_{t+1}(x, y)$ otherwise

where $H_{x y}=(1-\alpha) M B_{t}(x, y)+\alpha I_{t+1}(x, y), M B_{t}$ is the mean matrix of $B M_{1: t}$ at time $\mathrm{t}$ and $\alpha$ is a learning rate factor. Then, the tensor eigenspace model is updated incrementally and so on. The IRT show more robustness to noise than the IRPCA proposed by Li et al. [170]. 
Table 16. Performance evaluation on dynamic backgrounds and illumination changes

\begin{tabular}{|l|l|l|l|l|}
\hline Method & Dynamic backgrounds & Illumination changes & Indoor/outdoor scene & Applications \\
\hline \hline SG [13] & - & Slow changes & Indoor scene & Motion Capture \\
MOG [14] & Slow movement & Slow changes & Outdoor scene & Video Surveillance \\
KDE [15] & Yes & Slow changes & Outdoor scene & Video Surveillance \\
SL-PCA [165] & - & Yes & Outdoor scene (small objects) & Video Surveillance \\
\hline SVM [180] & - & Slow changes & Outdoor scene & Video Surveillance \\
SVR [183] & Slow movement & Slow changes & Outdoor scene & Video Surveillance \\
SVDD [189] & Yes & Yes & Outdoor scene & Video Surveillance \\
\hline SGG [190] & - & Slow changes & Indoor scene & Motion Capture \\
MOGG [194] & Slow movement & Slow changes & Outdoor scene & Video Surveillance \\
SL-ICA [200] & - & Yes & Outdoor scene (small objects) & Video Surveillance \\
SL-INMF [202] & - & Yes & Outdoor scene (small objects) & Video Surveillance \\
SL-IRT [205] & - & Yes & Outdoor scene (small objects) & Video Surveillance \\
\hline
\end{tabular}

Table 17. Computational complexity

\begin{tabular}{|l|l|l|l|}
\hline Method & Background Initialization & Background Maintenance & Foreground Detection \\
\hline \hline SG [13] & $\mathrm{O}(\mathrm{N})$ & $\mathrm{O}(1)$ & $\mathrm{O}(1)$ \\
MOG [14] & $\mathrm{O}(\mathrm{NK})$ & $\mathrm{O}(\mathrm{K})$ & $\mathrm{O}(\mathrm{K})$ \\
KDE [15] & $\mathrm{O}(\mathrm{N})$ & $\mathrm{O}(\mathrm{n})$ & $\mathrm{O}(1)$ \\
SL-PCA [165] & $\mathrm{O}(\mathrm{N})$ & $\mathrm{O}(\mathrm{N}+\mathrm{t})$ & $\mathrm{O}(\mathrm{P})$ \\
\hline SVM [180] & $\mathrm{O}(\mathrm{N})$ & $\mathrm{O}(1)$ & $\mathrm{O}(1)$ \\
SVR [183] & $\mathrm{O}(\mathrm{N})$ & $\mathrm{O}(1)$ & $\mathrm{O}(1)$ \\
SVDD [189] & $\mathrm{O}(\mathrm{N})$ & $\mathrm{O}(1)$ & $\mathrm{O}(1)$ \\
\hline SGG [190] & $\mathrm{O}(\mathrm{N})$ & $\mathrm{O}(\mathrm{K})$ & $\mathrm{O}(1)$ \\
MOGG [194] & $\mathrm{O}(\mathrm{NK})$ & $\mathrm{O}(\mathrm{M})$ & $\mathrm{O}(\mathrm{K})$ \\
SL-ICA [200] & $\mathrm{O}(\mathrm{N})$ & $\mathrm{O}(\mathrm{M})$ & $\mathrm{O}(\mathrm{P})$ \\
SL-INMF [202] & $\mathrm{O}(\mathrm{N})$ & $\mathrm{O}(\mathrm{M})$ & $\mathrm{O}(\mathrm{P})$ \\
SL-IRT [205] & $\mathrm{O}(\mathrm{N})$ & $\mathrm{O}(\mathrm{P})$ \\
\hline
\end{tabular}

\section{6 . PERFORMANCE EVALUATION}

We have firstly evaluated the ability of each method to deal with dynamics backgrounds and illumination changes. Then, the evaluation is conducted of per-pixel computational complexity and memory requirements.

\subsection{Challenges}

Table 16 groups the ability of each method to deal with dynamics backgrounds and illumination changes. The third column indicates in which type of scene the method is well suited. The related applications are indicated in the fourth column.

\subsection{Computational complexity}

The SG is the fastest method because the classification is just made using a threshold and the background maintenance just adapts the mean and the variance. Its complexity depends on $\mathrm{N}$ for the initialization. The MOG method has $\mathrm{O}(\mathrm{NK})$ complexity with $\mathrm{K}$ the number of Gaussian distributions used, typically between 3 and 5. For maintenance, the KDE computes its value in the Gaussian kernels centered on the past $\mathrm{n}$ frames, thus raising $\mathrm{O}(\mathrm{n})$ complexity, with $\mathrm{n}$ typically as high as 100 . For the reconstructive subspace learning, their computational complexities are related to the operations needed to compute the elements stored and updated, i.e the principal matrix or the eigenstructures. For example, the incremental tensor subspace learning requires $\mathrm{O}\left(\mathrm{I}_{1} \mathrm{I}_{2}\left(\mathrm{R}_{1}+\mathrm{R}_{2}\right.\right.$ $\left.+R_{3}\right)$ ) operations [205]. For the foreground detection, the reconstructive subspace learning methods have an estimated complexity per pixel of $\mathrm{O}(\mathrm{P})$, where $\mathrm{P}$ is the number of the best eigenvectors. For the background maintenance, their complexity is related to $\mathrm{M}$ which is the number of samples used to update the model. $\mathrm{M}=1$ if the model is update every frame. Table $\mathbf{1 7}$ shows the per-pixel computational complexity of each algorithm at each stage. More details about the complexity of each algorithm can be found in their corresponding papers.

\subsection{Memory requirements}

For the statistical methods, the memory complexity per pixel is the same as the computational complexity. At classification time, reconstructive approaches require a memory complexity per pixel $\mathrm{O}(\mathrm{P})$, with $\mathrm{P}$ the number of the best eigenvectors. However, at training time these methods require allocation of all the $\mathrm{N}$ training images, with an $\mathrm{O}(\mathrm{N})$ complexity. For the reconstructive subspace learning, the memory requirements are related to the elements stored and updated, i.e the principal matrix or the eigenstructures. For example, the incremental tensor subspace learning requires $\mathrm{O}\left(\mathrm{I}_{1} \mathrm{R}_{1}+\mathrm{I}_{2} \mathrm{R}_{2}+\left(\mathrm{I}_{1} \mathrm{I}_{2}\right) \mathrm{R}_{3}\right)$ memory units [205]. 


\section{COMPARISON}

We have chosen to compare different improvements of the MOG for dynamic backgrounds and the subspace learning models (SL-PCA, SL-ICA, SL-INMF and SL-IRT) for illumination changes. Results on the Wallflower dataset provided by Toyama et al. [20] are presented. We collected these results because of how frequent its use is in this field. This frequency is due to its faithful representation of real-life situations typical of scenes susceptible to video surveillance. Moreover, it consists of seven video sequences in which each sequence presenting one of the difficulties a practical task is likely to encounter (i.e illumination changes, dynamic backgrounds). The size of the images is $160 * 120$ pixels. A brief description of the Wallflower image sequences can be made as follows:

- Moved Object (MO): A person enters into a room, makes a phone call, and leaves. The phone and the chair are left in a different position. This video contains 1747 images

- Time of Day (TOD): The light in a room gradually changes from dark to bright. Then, a person enters the room and sits down. This video contains 5890 images

- Light Switch (LS): A room scene begins with the lights on. Then a person enters the room and turns off the lights for a long period. Later, a person walks in the room, switches on the light, and moves the chair, while the door is closed. This video contains 2715 images.

- Waving Trees (WT): A tree is swaying and a person walks in front of the tree. This video contains 287 images.

- Camouflage (C): A person walks in front of a monitor, which has rolling interference bars on the screen. The bars include similar color to the person's clothing. This video contains 353 images.

- Bootstrapping (B): The image sequence shows a busy cafeteria and each frame contains people. This video contains 3055 images.

- Foreground Aperture (FA): A person with uniformly colored shirt wakes up and begins to move slowly. This video contains 2113 images.

For each sequence, the ground truth is provided for one image when the algorithm has to show its robustness to a specific change in the scene. Thus, the performance is evaluated against hand-segmented ground truth. Three terms are used in the evaluation: False Positive (FP) is the number of background pixels that are wrongly marked as foreground; False Negative (FN) is the number of foreground pixels that are wrongly marked as background; Total Error (TE) is the sum of FP and FN.

\subsection{MOG and its improvements}

For the first category, we compare the MOG with its main improvements. Table $\mathbf{1 8}$ and Fig. (5) group the experimental results found in the literature for the algorithms chosen which are:

\section{The original algorithm: Stauffer and Grimson [14].}

2. Three intrinsic improvements: White et al. [67] which used a better setting for the learning rates using Particle Swarm Optimization, Wang et al. [60] which modified the foreground detection step using a mixed color space i.e a normalized RGB color space for pixels with high intensities and in RGB color space for pixels with low intensities and Setiawan et al. [97] which used the IHLS space.

3. Three extrinsic improvements: Schindler et al. [109] which used the MRFS to smooth the results spatially, Cristani et al. [117] which proposed the Spatial-Time Adaptive Per Pixel Mixture Of Gaussian called STAPPMOG and Cristani et al. [118] which used an adaptive spatio-temporal neighborhood analysis called ASTNA. For these two last algorithms, the authors don't give the result for the following image sequences: Moved Object, Time of Day and Light Switch. So, we have indicated for these the Total Error without these image sequences.

From Table 18, we can see that the original MOG gives the bigger total of error. A better setting of the learning rate and the threshold T using the PSO [67] divides approximately by 2 the number of total errors. The use of the IHLS color space [97] decreases a lot the number TE which becomes just under 10000 . The improvement proposed by Wang et al. [60] gives the better results for the intrinsic improvements. For the extrinsic improvements, the best results are obtained by MOG using MRF proposed by Schindler et al. [109] followed by S-TAPPMOG [117] and ASTNA [118]. For all the methods, the image sequences Light Switch (LS) gives the larger amount of false positive. Here, the best result is obtained by the method proposed by Schindler et al. [109]. The use of IHLS [97] gives it best improvement for the image sequences Camouflage (C) and for the method proposed by Wang et al. [30], it is the image sequences Waving Trees (WT). In conclusion, this performance evaluation shows that taking into account spatial and temporal consistency improves the results in a significant way. Fig. (6) presents the overall performance for the five first algorithms. It is not intended to be a definitive ranking of these algorithms. Such a ranking is necessarily task-, sequence-, and application dependent. 
Fig. (5). Results on the Wallflower dataset [26] for the MOG and its improvements.

\begin{tabular}{|c|c|c|c|c|c|c|c|}
\hline Sequence & $\mathrm{MO}$ & TD & LS & WT & $\mathrm{C}$ & $\mathrm{B}$ & $\mathrm{FA}$ \\
\hline Test image & & & & & & & \\
\hline Ground Truth & & & & & & & \\
\hline $\begin{array}{l}\text { MOG } \\
\text { Stauffer et al. [14] }\end{array}$ & & & & & & & \\
\hline $\begin{array}{l}\text { MOG with PSO } \\
\text { White } \text { et al. }[67]\end{array}$ & & & & & & & \\
\hline $\begin{array}{l}\text { MOG using IHLS } \\
\text { Setiawan et al. [97] }\end{array}$ & & & & & & & \\
\hline $\begin{array}{l}\text { Improved MOG } \\
\text { Wang et al. }[60]\end{array}$ & & & & & & & \\
\hline $\begin{array}{l}\text { MOG with MRF } \\
\text { Schindler } \text { et al. [109] }\end{array}$ & & & & & & & \\
\hline $\begin{array}{l}\text { S-TAPMOG } \\
\text { Cristani et al. [117] }\end{array}$ & - & - & - & & & & \\
\hline $\begin{array}{l}\text { ASTNA } \\
\text { Cristani et al. [118] }\end{array}$ & - & - & - & & & & \\
\hline
\end{tabular}

Table 18. Comparison on the Wallflower dataset [26] for the MOG and its improvements.

\begin{tabular}{|c|c|c|c|c|c|c|c|c|c|}
\hline \multirow[b]{2}{*}{ Algorithm } & & \multicolumn{7}{|c|}{ Problem Type } & \multirow[b]{2}{*}{ TE } \\
\hline & & $\mathrm{MO}$ & $\mathrm{TD}$ & LS & WT & $\mathrm{C}$ & $\mathrm{B}$ & FA & \\
\hline \multirow[t]{2}{*}{ MOG [14] } & $\mathrm{FN}$ & 0 & 1008 & 1633 & 1323 & 398 & 1874 & 2442 & \\
\hline & FP & 0 & 20 & 14169 & 341 & 3098 & 217 & 530 & 27053 \\
\hline \multirow[t]{2}{*}{ MOG with PSO [67] } & $\mathrm{FN}$ & 0 & 807 & 1716 & 43 & 2386 & 1551 & 2392 & \\
\hline & FP & 0 & 6 & 772 & 1689 & 1463 & 519 & 572 & 13916 \\
\hline \multirow[t]{2}{*}{ MOG-IHLS [97] } & $\mathrm{FN}$ & 0 & 379 & 1146 & 31 & 188 & 1647 & 2327 & \\
\hline & FP & 0 & 99 & 2298 & 270 & 467 & 333 & 554 & 9739 \\
\hline \multirow[t]{2}{*}{$\begin{array}{l}\text { Improved MOG - FD } \\
\text { [60] }\end{array}$} & $\mathrm{FN}$ & 0 & 597 & 1481 & 44 & 106 & 1176 & 1274 & \\
\hline & FP & 0 & 358 & 669 & 288 & 413 & 134 & 541 & 7081 \\
\hline \multirow[t]{2}{*}{ MOG with MRF [109] } & $\mathrm{FN}$ & 0 & 47 & 204 & 15 & 16 & 1060 & 34 & \\
\hline & FP & 0 & 402 & 546 & 311 & 467 & 102 & 604 & 3808 \\
\hline \multirow[t]{2}{*}{ S-TAPPMOG [117] } & $\mathrm{FN}$ & - & - & - & 153 & 643 & 1414 & 1912 & \\
\hline & FP & - & - & - & 1152 & 1382 & 811 & 377 & 7844 \\
\hline \multirow[t]{2}{*}{ ASTNA [118] } & $\mathrm{FN}$ & - & - & - & 253 & 823 & 2349 & 1900 & \\
\hline & FP & - & - & - & 100 & 1173 & 73 & 360 & 7031 \\
\hline
\end{tabular}


Fig. (7). Results on the Wallflower dataset [26] for the subspace learning models.

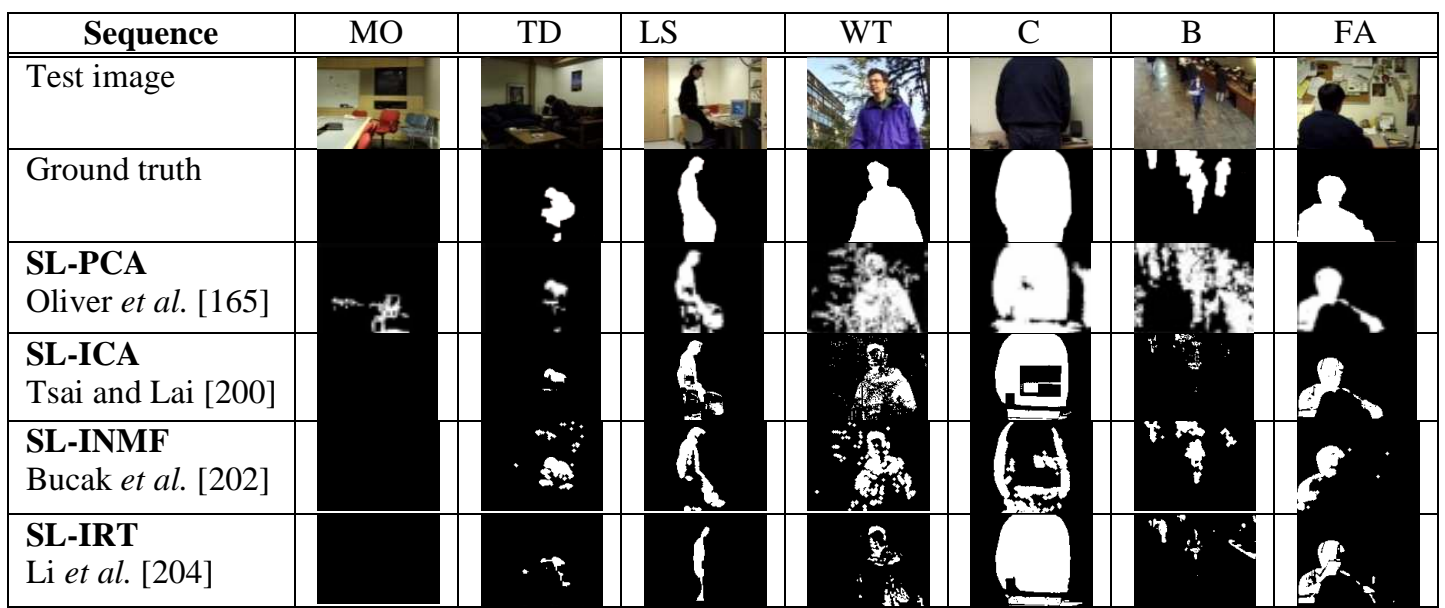

Table 19. Comparison on the Wallflower dataset [26] for the subspace learning models.

\begin{tabular}{|c|c|c|c|c|c|c|c|c|c|}
\hline \multirow[b]{2}{*}{ Algorithm } & & \multicolumn{7}{|c|}{ Problem Type } & \multirow[b]{2}{*}{ TE } \\
\hline & & $\mathrm{MO}$ & TD & $\mathrm{LS}$ & WT & $\mathrm{C}$ & B & FA & \\
\hline \multirow[t]{2}{*}{ SL-PCA [165] } & FN & 0 & 879 & 962 & 1027 & 350 & 304 & 2441 & 17677 \\
\hline & FP & 1065 & 16 & 362 & 2057 & 1548 & 6129 & 537 & \\
\hline \multirow[t]{2}{*}{ SL-ICA [200] } & FN & 0 & 1199 & 1557 & 3372 & 3054 & 2560 & 2721 & 15308 \\
\hline & FP & 0 & 0 & 210 & 148 & 43 & 16 & 428 & \\
\hline \multirow[t]{2}{*}{ SL-INMF [202] } & FN & 0 & 724 & 1593 & 3317 & 6626 & 1401 & 3412 & 19098 \\
\hline & FP & 0 & 481 & 303 & 652 & 234 & 190 & 165 & \\
\hline \multirow[t]{2}{*}{ SL-IRT [204] } & FN & 0 & 1282 & 2822 & 4525 & 1491 & 1734 & 2438 & 17053 \\
\hline & FP & 0 & 159 & 389 & 7 & 114 & 2080 & 12 & \\
\hline
\end{tabular}

\subsection{Subspace learning models}

SL-PCA which is from the first category is compared with the subspace learning models from the third category: SLIRT, SL-PCA and SL-INMF. Table 19 and Fig. (7) group the experimental results found in the literature for the subspace learning algorithms. From Table 19, we can see that SL-ICA gives the smallest TE followed by SL-IRT, SL-PCA and SLINMF. Fig. (8) shows the overall performance. This ranking has to be taken with precaution because a poor performance on one video influences the TE and then modifies the rank. The main interpretation is that all the models are robust to illumination changes as can be seen on the sequence called "Time of Day" (TD) and "Light Switch" (LS). Otherwise, the subspace learning algorithms are more or less adapted for specific situations. For example, only SL-PCA gives FP in the sequence called "Moved Objects" (MO) due the fact that the model is not update overtime. In the same way, SLINMF gives the biggest total error due to its results on the sequence called "Camouflage" $(\mathrm{C})$. This is confirmed by the Fig. (9). which shows the performance without this sequence. In this case, SL-INMF is the second in term of performance. SL-ICA has globally good performance except for the sequence called "Bootstrap" (B) by giving less true detection.
SL-IRT seems to be more efficient in the case of camouflage. SL-PCA gives less FN than FP. For SL-ICA, SL-INMF and SL-IRT, it is the contrary. We can remark that SL-ICA provides very less FP than FN. It is interesting in videosurveillance because it decreases false alarms.

Fig. (6). Overall performance on the Wallflower dataset [26] for the MOG and its improvements.

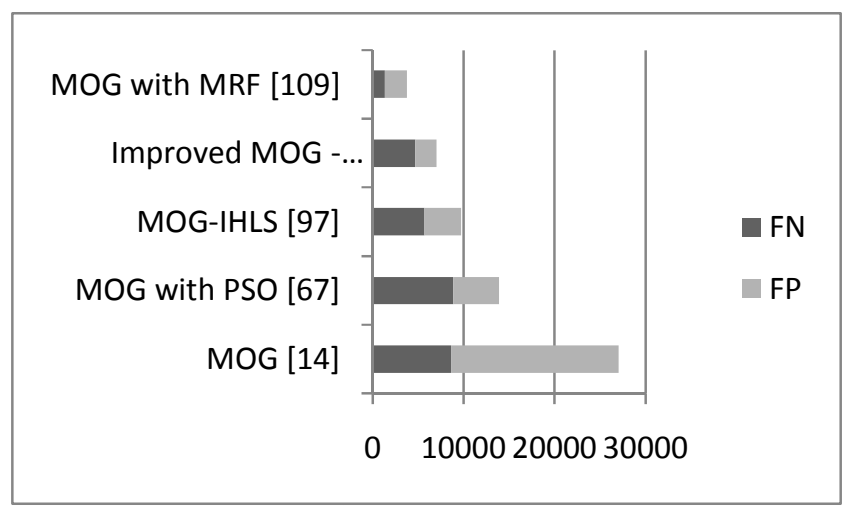


Fig. (8). Overall performance on the Wallflower dataset [26] for the subspace learning models.

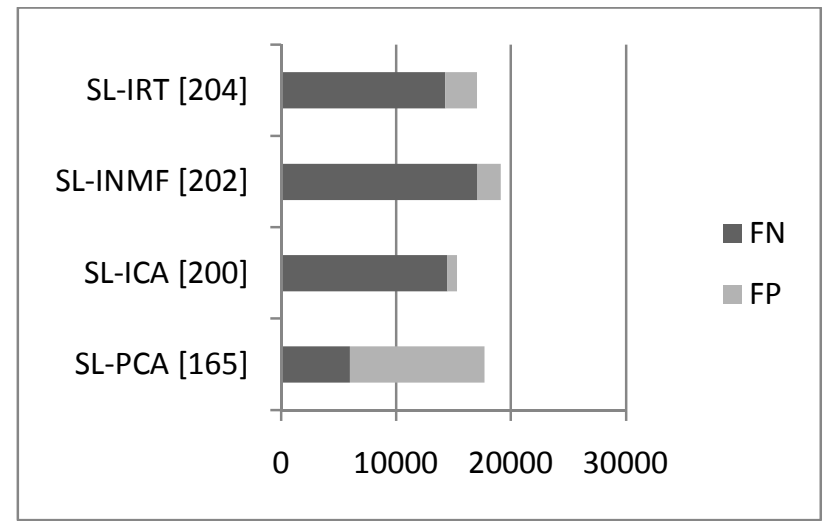

Fig. (9). Overall performance on the Wallflower dataset [26] without the sequence called "Camouflage" for the subspace learning models.

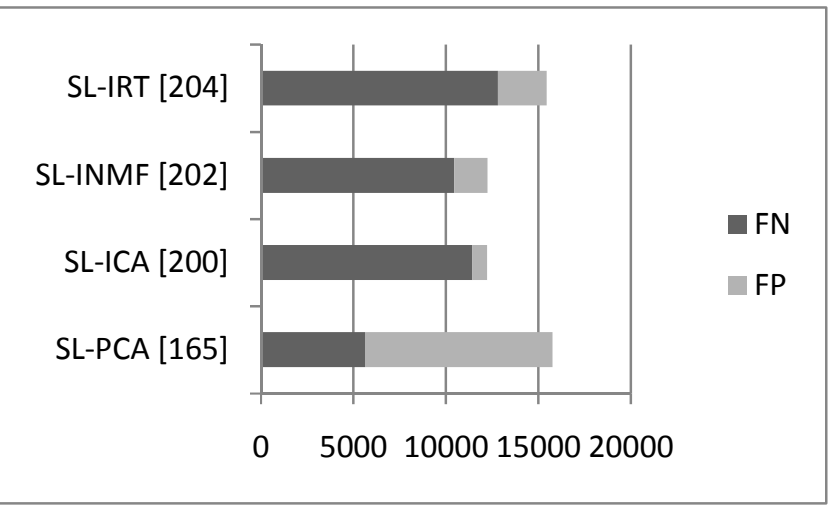

\section{CURRENT \& FUTURE DEVELOPMENTS}

This paper attempts to provide a comprehensive survey on statistical background modeling for foreground detection and to provide some structural categories for the strategies developed in 300 papers and 10 recent patents. Thus, we proposed a classification in term of category. For the MOG and $\mathrm{KDE}$, we proposed a classification for their related improvements in two classes respectively called intrinsic and extrinsic improvements. Strategies adding spatial and temporal information in the different steps or in added process proved their abilities to improve the robustness of the original model to the critical situations. Cooperation with other segmentations has shown their interests too. Methods which reduce the computation time permit to deal with the constraints of real-time application. Although significant progress has been made, there is still work to be done and we believe that a systematic comparative evaluation must be made and thus determine the best combination of strategies. In this context, we encourage the evaluation using the Wallflower dataset like in $[60,67,97,109]$.
Furthermore, two main investigations seem to be very promising:

- For dynamic backgrounds, combination between SG, MOG and KDE [337-339] which allows to gives more robustness when there are waving trees, water surfaces and water rippling in the scene.

- For illumination changes, robust PCA [340-344] in which the background is modeled by a low rank subspace that can gradually change over time, while the moving foreground objects are considered as the correlated sparse outliers.

In conclusion, this paper allows the reader to survey recent advances on statistical background modeling and it can effectively guide him to select the best improvement for his specific application. Particularly, this survey paper allows: 1) Developers to choose the appropriate improvement to tackle the critical situations met in their application. 2) Researchers to have a recent state-of-the-art and so easily identify new ideas. 3) Reviewers to verify quickly the originality of a paper.

\section{ACKNOWLEDGMENTS}

The authors would like to thanks Serhat Selcuk BUCAK (Pattern Recognition and Image Processing Lab, Michigan State University, USA) who provided the results obtained by his algorithm SL-INMF, Du-Ming TSAI and Shia-Chih LAI (Department of Industrial Engineering and Management, Yuan-Ze University, Taiwan) who provided the results obtained by their algorithm SL-ICA.

\section{CONFLICT OF INTEREST}

The author declared no conflict of interest

\section{REFERENCES}

[1] Cheung S., Kamath C. Robust Background Subtraction with Foreground Validation for Urban Traffic Video, Journal of Applied Signal Processing, Special Issue on Advances in Intelligent Vision Systems: Methods and Applications, EURASIP 2005, Issue 14, pages 2330-2340, New York, USA, 2005.

[2] Carranza A., Theobalt C., Magnor M., Seidel H. Free-Viewpoint Video of Human Actors, ACM Transactions on Graphics, Volume 22, Issue 3, pages 569-577, 2003.

[3] Horprasert T., Haritaoglu I., Wren C., Harwood D., Davis L. Pentland A., Real-time 3D Motion Capture, Workshop on Perceptual User Interfaces, PUI 1998, San Francisco, California, pages 87-90, November 1998.

[4] Mikic I., Trivedi M., Hunter E., Cosman P. Human Body Model Acquisition and Tracking using Voxel Data, International Journal of Computer Vision, pages 199-223, July 2003.

[5] El Baf F., Bouwmans T., Vachon B. Comparison of Background Subtraction Methods for a Multimedia Learning Space, International Conference on Signal Processing and Multimedia, SIGMAP 2007, Barcelona, Spain, July 2007.

[6] Pande A., Verma A., Mittal A. Network aware optimal resource allocation for e-learning Videos, International conference on mobile Learning, Melbourne Australia, October 2007.

[7] Warren J., Unencumbered Full Body Interaction in Video Games, Thesis, MFA Design and Technology Parsons School of Design, New York, USA, April 2003.

[8] Elhabian S., El-Sayed K., Ahmed S. Moving Object Detection in Spatial Domain using Background Removal Techniques - State-of-Art, 
Recent Patents on Computer Science, Volume 1, Number 1, pages 3254, January 2008.

[9] Cristani M., Farenzena M., Bloisi D., Murino V. Background Subtraction for Automated Multisensor Surveillance: A Comprehensive Review, EURASIP Journal on Advances in Signal Processing, 24 pages, Volume 2010, 2010.

[10] Lee B., Hedley M. Background Estimation for Video Surveillance, , IVCNZ 2002, pages 315-320, 2002.

[11] McFarlane N., Schofield C. Segmentation and tracking of piglets in images, BMVA 1995, pages 187-193, 1995.

[12] Zheng J., Wang Y., Nihan N., Hallenbeck, E. Extracting Roadway Background Image: A mode based approach, Journal of Transportation Research Report, No 1944, pages 82-88, March 2006.

[13] Wren C., Azarbayejani A., Darrell T., Pentland A. Pfinder: Real-Time Tracking of the Human Body, IEEE Transactions on Pattern Analysis and Machine Intelligence, Volume 19, No. 7, pages 780-785, July 1997.

[14] Stauffer C., Grimson W. Adaptive background mixture models for real-time tracking, CVPR 1999, pages 246-252, 1999.

[15] Elgammal A., Harwood D., Davis L. Non-parametric Model for Background Subtraction, ECCV 2000, pages 751-767, Dublin, Ireland, June 2000 .

[16] Sigari M., Mozayani N., Pourreza H. Fuzzy Running Average and Fuzzy Background Subtraction: Concepts and Application, International Journal of Computer Science and Network Security, Volume 8, No. 2, pages 138-143, 2008.

[17] El Baf F., Bouwmans T., Vachon B., Type-2 fuzzy mixture of Gaussians model: Application to background modeling, ISVC 2008, pages 772-781, Las Vegas, USA, December 2008.

[18] Zhang H., Xu D Fusing Color and Texture Features for Background Model, International Conference on Fuzzy Systems and Knowledge Discovery, Volume 4223, No. 7, pages 887-893, September 2006.

[19] El Baf F., Bouwmans T., Vachon B., "Fuzzy Integral for Moving Object Detection”, FUZZ-IEEE 2008, pages 1729-1736, Hong-Kong, China, June 2008.

[20] Toyama K., Krumm J. Brumitt B., Meyers B. Wallflower: Principles and Practice of Background Maintenance, International Conference on Computer Vision, pages 255-261, Corfu, Greece, September 1999.

[21] Messelodi S., Modena C., Segata N., Zanin M. A Kalman filter based background updating algorithm robust to sharp illumination changes, ICIAP 2005, Volume 3617, pages 163-170, Cagliari, Italy, September 2005.

[22] Chang R., Ghandi T., Trivedi M., Vision modules for a multi sensory bridge monitoring approach, ITSC 2004, pages 971-976, October 2004.

[23] Wang H., Suter D. A Novel Robust Statistical Method for Background Initialization and Visual Surveillance, ACCV 2006, Hyderabad, India, Volume 3851, pages 328-337, January 2006.

[24] Porikli F. Human Body Tracking by Adaptive Background Models and Mean-Shift Analysis, PETS 2003, March 2003.

[25] Porikli F., Tuzel O., "Bayesian Background Modeling for Foreground Detection", VSSN 2005, pages 55-28, November 2005.

[26] Wallflower Dataset: http://research.microsoft.com/users/jckrumm/WallFlower/TestImages. $\underline{\mathrm{htm}}$

[27] ATON Dataset: http://cvrr.ucsd.edu/aton/shadow/

[28] OTCBVS Dataset: http://www.cse.ohio-state.edu/otcbvs-bench/

[29] VSSN Dataset: http://mmc36.informatik.uni-augsburg.de/VSSN06_OSAC/

[30] PETS Dataset: http://pets2006.net/

[31] IPPR Dataset: http://archer.ee.nctu.edu.tw/contest/

[32] Davis J., Goadrich M. The Relationship between Precision-Recall and ROC Curves, ICML 2006, Volume 148, pages 233-240, Pittsburgh, Pennsylvania, 2006.

[33] Kim K., Chalidabhongse T., Harwood D., Davis L. PDR: Performance Evaluation Method for Foreground-Background Segmentation Algorithms, EURASIP Journal on Applied Signal Processing, 2006.

[34] Li L., Huang W., Gu I., Tian Q. Statistical Modeling of Complex Background for Foreground Object Detection, IEEE transaction image processing, Volume 13, Issue 11, pages 1459-1472, November 2004.

[35] Nascimento J., Marques J. Performance Evaluation of Object Detection Algorithms for video Surveillance, IEEE Transaction on Multimedia, pages 761-774, August 2006
[36] Bhaskar H., Mihaylova L., Maskell S. Automatic Target Detection Based on Background Modeling Using Adaptive Cluster Density Estimation, German Workshop on Sensor Data Fusion: Trends, Solutions, Applications, Universität Bremen, Germany, September 2007

[37] Han B., Lin X. Update the GMMs via adaptive Kalman filtering, Proceedings of SPIE, Volume 5960, Issue 3, pages 1506-1515, 2005.

[38] Yang H. Tan Y., Tian J., Liu J., "Accurate dynamic scene model for moving object detection”, ICIP 2007, Volume VI, pages 157-160, 2007.

[39] Zhang W., Fang X., Yang X., Wu Q. Spatiotemporal Gaussian mixture model to detect moving objects in dynamic scenes, Journal of Electronic Imaging, Volume 16, Issue 2, April 2007.

[40] Tang P., Gao L., Liu Z. Salient Moving Object Detection Using Stochastic Approach Filtering, ICIG 2007, pages 530-535, 2007.

[41] Harville M. A framework for high-level feedback to adaptive, perpixel, mixture-of-Gaussian background models, ECCV 2002, pages 543 -560, Copenhagen, Denmark, May 2002.

[42] Cristani M., Murino V.A spatial sampling mechanism for effective background subtraction, VISAPP 2007, Volume 2, pages 403-410, Barcelona, Spain, March 2007.

[43] François A., Medioni G. Adaptive Color Background Modeling for Real-time Segmentation of Video Streams, International Conference on Imaging Science, Systems, and Technology, pages 227-232, Las Vegas, Nevada, June 1999.

[44] Zhao M., Li N., Chen C., Robust automatic video object segmentation technique, International Conference on Image Processing, September 2002.

[45] Friedman N., Russell S. Image Segmentation in Video Sequences: A Probabilistic Approach, UAI 1997, pages 175-181, 1997.

[46] Atev S., Masoud O., Papanikolopoulos N. Practical Mixtures of Gaussians with Brightness Monitoring, ITS 2004, pages 423-428, 2004.

[47] Zang Q., Klette R., Parameter Analysis for Mixture of Gaussians, CITR Technical Report 188, Auckland University, 2006.

[48] Bouwmans T., El Baf F. Vachon B., Background Modeling using Mixture of Gaussians for Foreground Detection - A survey, Recent Patents on Computer Science, Volume 1, No 3, pages 219-237, November 2008.

[49] Zivkovic Z. Improved adaptive Gaussian mixture model for background subtraction, International Conference Pattern Recognition, Volume 2, pages 28-31, 2004.

[50] Cheng J., Yang J., Zhou Y., Cui Y. Flexible background mixture models for foreground segmentation, Image and Vision Computing, Volume 24, pages 473-482, 2006.

[51] Shimada A., Arita D., Taniguchi R. Dynamic Control of Adaptive Mixture-of-Gaussians Background Model, AVSS 2006, page 5, Sydney, Australia, November 2006.

[52] Tan R., Huo H., Qian J., Fang T. Traffic Video Segmentation using Adaptive-K Gaussian Mixture Model, IWICPAS 2006, LNCS 4153 , pages125-134, Xi'An, China, August 2006.

[53] Carminati L., Benois-Pinau J. Gaussian Mixture Classification for Moving Object Detection in Video Surveillance Environment, ICIP 2005, 2005.

[54] Morellas V., Pavlidis L., Tsiamyrtzis P. DETER: detection of events for threat evaluation and recognition, Machine Vision and Applications, Volume 15, pages 29-45, June 2003.

[55] Lee D. Online Adaptive Gaussian Mixture Learning for Video Applications, ECCV Workshop on Statistical Methods for Video Processing, Prague, Czech, May 2004.

[56] Zhang Y., Liang Z., Hou Z., Wang H., Tan M. An Adaptive Mixture Gaussian Background Model with Online Background Reconstruction and Adjustable Foreground Mergence Time for Motion Segmentation, ICIT 2005, pages 23-27, December 2005.

[57] Amintoosi M., Farbiz F., Fathy M., Analoui M., Mozayani N. QR decomposition-based algorithm for background subtraction, ICASSP 2007, 2007.

[58] Lepisk A. The use of Optic Flow within Background Subtraction, Master Thesis, Royal Institute of Technology, Nada, Sweden, January 2005.

[59] Han B., Lin X., Update the GMMs via adaptive Kalman filtering, Proceedings of SPIE, Volume 5960, Issue 3, pages 1506-1515, 2005. 
[60] Wang H., Suter D. A Re-Evaluation of Mixture-of-Gaussian Background Modeling, ICASSP 2005, Pennsylvania, USA, pages 1017-1020, March 2005 .

[61] Lindstrom J., Lindgren F., Ltrstrom K., Holst J., Holst U., Background and Foreground Modeling Using an Online EM Algorithm, VS 2006 in conjunction with ECCV 2006, May 2006.

[62] Stauffer C., Grimson E. Learning Patterns of Activity Using RealTime Tracking, PAMI 2000, Volume 22, pages 747-757, 2000.

[63] Landabaso J., Pardas M. Cooperative Background Modelling using Multiple Cameras Towards Human Detection in Smart-Rooms, EUSIPCO 2006, Florence, Italy, September 2006.

[64] Park D., Kim J., Kim J., Cho S., Chung S. Motion Detection in Complex and Dynamic Backgrounds, PSIVT 2006, pages 545-552, Hsinchu, Taiwan, December 2006.

[65] Mittal A., Huttenlocher D. Scene Modeling for Wide Area Surveillance and Image Synthesis, CVPR 2000, Volume 2, pages 160167, Hilton Head, South Carolina, June 2000.

[66] Zang Q., Klette R., Evaluation of an Adaptive Composite Gaussian Model in Video Surveillance, CITR Technical Report 114, Auckland University, August 2002.

[67] White B., Shah M., Automatically Tuning Background Subtraction Parameters Using Particle Swarm Optimization, ICME 2007, pages 1826-1829, Beijing, China, 2007.

[68] KaewTraKulPong P., Bowden R. An Improved Adaptive Background Mixture Model for Real-time Tracking with Shadow Detection, AVBS 2001, Kingston, UK, September 2001.

[69] KaewTraKulPong P., Bowden R. Adaptive Visual System for Tracking Low Resolution Color Targets, BMVC 2001, Volume 1, pages 243-252, Manchester UK, September 2001.

[70] KaewTraKulPong P., Bowden R. A Real-Time Adaptive Visual Surveillance System for Tracking Low Resolution Color Targets In Dynamically Changing Scenes, Journal of Image and Vision Computing, Volume 21, Issue 10, pages 913-929, September 2003.

[71] Lee D. Improved Adaptive Mixture Learning for Robust Video Background Modeling, IAPR Workshop on Machine Vision for Applications, Nara, Japan, pages 443-446, December 2002

[72] Harville M., Gordon G., Woodfill J. Foreground segmentation using adaptive mixture models in color and depth, Workshop on Detection and Recognition of Events in Video, Vancouver, Canada, July 2001.

[73] Porikli F. Human Body Tracking by Adaptive Background Models and Mean-Shift Analysis, PETS 2003, March 2003.

[74] Liu Y., Ai H., Xu G. Moving object detection and tracking based on background subtraction, Proceedings of SPIE, Volume 4554, pages 62-66, 2001.

[75] Pnevmatikakis A., Polymenakos L. 2D Person Tracking Using Kalman Filtering and Adaptive Background Learning in a Feedback Loop, CLEAR Workshop 2006, LNCS 4122, pages 151-160, 2006.

[76] Stergiou A., Pnevmatikakis A., Polymenakos L. The AIT Outdoor tracker for Vehicle and Pedestrians in CLEAR 2007, CLEAR Workshop 2007, pages 148-159, 2007.

[77] Power P., Schoonees J. Understanding Background Mixture Models for Foreground Segmentation, Imaging and Vision Computing New Zealand, Auckland, NZ, November 2002.

[78] Leotta M., Mundy J. Learning Background and Shadow Appearance with 3-D Vehicle Models, BMVC 2006, Edimburgh, September 2006.

[79] Ren Y., Chua C., Ho Y. Motion Detection with Non-stationary Background, ICIAP 2002, pages 78-83, 2002.

[80] Lee D. Improved Adaptive Mixture Learning for Robust Video Background Modeling, IAPR Workshop on Machine Vision for Applications, Nara, Japan, pages 443-446, December 2002

[81] Sun Y. Better Foreground Segmentation for Static Cameras via New Energy Form and Dynamic Graph-cut, ICPR 2006, 2006.

[82] Morellas V., Pavlidis I., Tsiamyrtzis P. DETER: detection of events for threat evaluation and recognition, Machine Vision and Applications, Volume 15, pages 29-45, June 2003.

[83] Landabaso J., Pardas M., Xu L. Hierarchical Representation of Scenes using Activity Information, ICASSP 2005, pages 677-680, Philadelphia, USA, March 2005.

[84] Yang S., Hsu C. Background Modeling from GMM Likelihood Combined with Spatial and Color Coherency, ICIP 2006, 2006

[85] Lee D. Effective Gaussian Mixture Learning for Video Background Subtraction, PAMI 2005, Volume 27, pages 827-832 2005.

[86] Withagen P., Groen F., Schutte K. EMswitch: a multi-hypothesis approach to EM background modeling, ACIVS 2003, September 2003.
[87] Haque M., Murshed M., Paul M. A Hybrid Object Detection Technique from Dynamic Background Using Gaussian Mixture Models, IEEE International Workshop on Multimedia Signal Processing, MMSP 2008, pages 915-920, Cairns, Queensland, Australia, October 2008

[88] Haque M., Murshed M., Paul M. Improved Gaussian Mixtures for Robust Object Detection by Adaptive Multi-Background Generation, ICPR 2008, Tampa, Florida, USA, December 2008.

[89] Haque M., Murshed M., Paul M. On Stable Dynamic Background Generation Technique using Gaussian Mixture Models for Robust Object Detection, AVSS 2008, pages 41-48, Santa Fe, USA, September 2008.

[90] Fang X., Xiong W., Hu B., Wang L. A Moving Object Detection Algorithm Based on Color Information, IST 2006, Journal of Physics, Volume 48, pages 384-387, 2006.

[91] Pokrajac D., Latecki L. Spatiotemporal Blocks-Based Moving Objects Identification and Tracking, VS-PETS 2003, pages 70-77, Octobe 2003.

[92] Bhaskar H., Mihaylova L., Maskell S. Automatic Target Detection Based on Background Modeling Using Adaptive Cluster Density Estimation, LNCS from the 3rd German Workshop on Sensor Data Fusion: Trends, Solutions, Applications, Universität Bremen, Germany, September 2007.

[93] Stijnman G., van den Boomgaard R. Background estimation in video sequences, Technical Report 10, Intelligent Sensory Information Systems Group, University of Amsterdam, January 2000

[94] Xu M., Ellis T. Illumination-invariant motion detection using color mixture models, BMVA, Manchester, pages 163-172, September 2001.

[95] Wang W., Wu R. Fusion of luma and chroma GMMs for HMM-based object detection, Pacific Rim Symposium on Advances in Image and Video Technology, pages 573-81, Hsinchu, Taiwan, December 2006.

[96] Yang S., Hsu C. Background Modeling from GMM Likelihood Combined with Spatial and Color Coherency, ICIP 2006, pages 28012804, Atlanta, USA, 2006.

[97] Setiawan N., Hong S., Kim J., Lee C. Gaussian Mixture Model in Improved HLS Color Space for Human Silhouette Extraction, ICAT 2006, pages 732-741, Hangzhou, China, 2006.

[98] Kristensen F., Nilsson P., Öwall V. Background Segmentation Beyond RGB, ACCV 2006, pages 602-612, Hyderabad, Indian, 2006.

[99] Ribeiro H., Gonzaga A. Hand Image Segmentation in Video Sequence by GMM: a comparative analysis, SIBGRAPI 2006, pages 357-364, Brazil, October 2006.

[100]Javed O., Shafique K., Shah M. A Hierarchical Approach to Robust Background Subtraction using Color and Gradient Information, WMVC 2002, page 22, Orlando, December 2002.

[101] Jain V., Kimia B. , Mundy J. Background modelling based on subpixel edges, ICIP 2007, Volume VI, pages 321-324, San Antonio, USA, September 2007.

[102]Tian Y. , Hampapur A. Robust Salient Motion Detection with Complex Background for Real-time Video Surveillance, CVPR 2005, Volume 2, pages 30-35, Breckenridge, Colorado, January 2005.

[103] Gordon G., Darrell T., Harville M., Woodfill J. Background estimation and removal based on range and color, IEEE Conference on Computer Vision and Pattern Recognition, volume 2, pages 459-464, June 1999.

[104]Silvestre D. "Video surveillance using a time-of-flight camera, $\mathrm{PhD}$ thesis, Informatics and Mathematical Modelling, Technical University of Denmark, 2007.

[105]Dickinson P, Hunter A. Scene Modeling Using an Adaptive Mixture of Gaussians in Color and Space, IEEE Conference on Advanced Video and Signal based Surveillance, Como, Italy, September 2005.

[106]Wang W., Gao W., Yang J., Chen D. Modeling Background from Compressed Video, ICCV 2005, pages 161-168, Beijing, China, October 2005.

[107]Kumar P., Sengupta K. Foreground background segmentation using temporal and spatial markov processes, Department of Electrical and Computer Engineering, National University of Singapore, November 2000.

[108]Zhou D., Zhang H. Accurate Segmentation of Moving Objects in Image Sequence Based on Spatio-Temporal Information, ICMA 2006 , pages 543-548, Luoyang, China, June 2006.

[109] Schindler K, Wang H. Smooth Foreground-Background Segmentation for Video Processing, ACCV 2006, Hyderabad, India, Volume 3852, pages 581-590, January 2006. 
[110]Sun Y., Yuan B. Hierarchical GMM to handle sharp changes in moving object detection, Electronic Letters, Volume 40, No 13, pages 801-802, June 2004.

[111]Park J., Tabb A., Kak A. Hierarchical Data Structure for Real Time Background Subtraction, ICIP 2006, pages 1849-1852, Atlanta, USA, October 2006

[112]Chen Y., Chen C., Huang C., Hung Y. Efficient hierarchical method for background subtraction, Pattern Recognition, Volume 40, Issue 10, pages 2706-2715, October 2007.

[113]Zhou Y., Gong Y., Tao H. Background modeling using time dependent Markov random field with image pyramid, IEEE Motion 2005, January 2005.

[114]Zang Q., Klette R., "Robust Background Subtraction and Maintenance”, ICPR 2004, Volume 2, pages 90-93, 2004.

[115]Zhong Q., Dai L., Song Y., Wang R. A hierarchical motion detection algorithm with the fusion of the two types of motion information, Pattern Recognition and Artificial Intelligence, Volume 18, Issue 5, pages 552-557, October 2005.

[116]Cristani M., Bicego M., Murino V. Integrated Region- and Pixel-based Approach to Background Modeling, MOTION 2002, pages 3-8, 2002.

[117]Cristani M., Murino V. A spatial sampling mechanism for effective background subtraction, VISAPP 2007, Volume 2, pages 403-410, Barcelona, Spain, March 2007.

[118]Cristani M. , Murino V. Background subtraction with adaptive spatiotemporal neighborhood analysis, VISAPP 2008, Funchal, Portugal, January 2008.

[119]Su T., Hu J. Background Removal in Vision Servo System using Gaussian Mixture Model Framework, ICNSC 2004, Volume 1, pages 70-75, March 2004.

[120]Hu J., Su T. Robust Background Subtraction with Shadow and Highlight Removal for Indoor Surveillance, Journal on Advances in Signal Processing, Volume 2007, pages 1-14, 2007.

[121]Porikli F. Detection of Temporarily Static regions by Processing Video at Different Frame Rates, AVSS 2007, pages 236-241, 2007.

[122] Yang H., Tan Y., Tian J., Liu J. Accurate dynamic scene model for moving object detection, ICIP 2007, Volume 6, pages 157-160, 2007.

[123]Porikli F., Tuzel O. Bayesian Background Modeling for Foreground Detection, VSSN 2005, pages 55-28, November 2005

[124]Xu M., Ellis T. Color-invariant motion detection under fast illumination changes, IAPR European Workshop on Advanced Videobased Surveillance Systems, Kingston, pages 335-345, September 2001.

[125]Nadimi S., Bhanu B. Multistrategy fusion using mixture model for moving object detection, International Conference on Multisensor Fusion and Integration for Intelligent Systems, pages 317-322, BadenBaden, Germany, August 2001.

[126]Nadimi S., Bhanu B. Physics-based cooperative sensor fusion for moving object detection, IEEE Workshop on Learning in Computer Vision and Pattern Recognition, Washington, DC, June 2004.

[127]Conaire C., O'Connor N., Cooke E. , Smeaton A. Multispectral Object Segmentation and Retrieval in Surveillance Video, ICIP 2006, pages 2381-2384, 2006.

[128] Harville M. A framework for high-level feedback to adaptive, perpixel, mixture-of-Gaussian background models, European Conference on Computer Vision, Copenhagen, Denmark, May 2002.

[129]Taycher L., Fisher J., Darrell T. Incorporating Object Tracking Feedback into Background Maintenance Framework, IEEE Workshop on Motion and Video Computing, Volume 2, pages 120-125, 2005

[130]Turdu D., Erdogan H. Improved post-processing for GMM based adaptive background modeling, ISCIS 2007, pages 1-6, November 2007.

[131]Parks D., Fels S., Evaluation of Background Subtraction Algorithms with Post-processing, AVSS 2008, September 2008.

[132] Atrey P., Kumar V., Kumar A., Kankanhalli M. Experiential sampling based foreground/background segmentation for video surveillance, ICME 2006, pages 1809-1812, Toronto, Canada, July 2006.

[133] Magee D. Tracking Multiple Vehicles using Foreground, Background and Motion Models, Image and Vision Computing, volume 22, pages 143-155, 2004

[134]Zuo J., Pan Q., Liang Y., Zhang H., Cheng Y. Model Switching Based Adaptive Background Modeling Approach, Acta Automatica Sinica, Volume 33, Issue 5, pages 467-473, 2007.

[135]Tang Z. , Miao Z. Fast Background Subtraction Using Improved GMM and Graph Cut”, CISP 2008, Sanya, Hainan, China, May 2008.
[136]Jiang H., Ardo H., Owall V. Hardware accelerator design for video segmentation with multi-modal background modeling, ISCAS 2005, Volume 2, pages 1142- 1145, May 2005.

[137]Appiah K., Hunter A. A Single-Chip FPGA Implementation of Realtime Adaptive Background Model, IEEE 2005 Conference on FieldProgrammable Technology, FPT 2005, National University of Singapore, Singapore, December 2005

[138]Al-Mazeed A., Nixon M., Gunn S. Classifiers Combination for Improved Motion Segmentation, ICIAR 2004, pages 363-371, Porto, Portugal, 2004.

[139]Fang X., Xiong W., Hu B., Wang L., “A Moving Object Detection Algorithm Based on Color Information, Journal of Physics, Volume 48, pages 384-387, 2006.

[140]Zhang J., Chen C. Moving Objects Detection and Segmentation in Dynamic Video Backgrounds, Conference on Technologies for Homeland Security, pages 64-69, Woburn, USA, May 2007.

[141]Tavakkoli A. Automatic Video Object Plane Extraction using nonParametric Kernel Density Estimation, Mathematical Methods in Computer Vision, University of Nevada, Reno, NV, May 2005.

[142]Tavakkoli A., Nicolescu M, G. Bebis G. "Automatic Statistical Object Detection for Visual Surveillance, SSIAI 2006, March 2006.

[143]Ianasi C., Gui V., Toma C, Pescaru D. A Fast Algorithm for Background Tracking in Video Surveillance, Using Nonparametric Kernel Density Estimation”, Facta Universitatis, Series: Electronics and Energetics, Volume 18, No. 1, pages 127-144, April 2005.

[144]Tanaka T., Shimada A., Arita D., Taniguchi R. Non-parametric Background and Shadow Modeling for Object Detection, ACCV 2007, pages 159-168, Tokyo, Japan, November 2007

[145]Tanaka T., Shimada A., Arita D., Taniguchi R. A Fast Algorithm for Adaptive Background Model Construction Using Parzen Density Estimation, AVSS 2007, pages 528-533, London, UK, September 2007.

[146]Zivkovic Z. Efficient adaptive density estimation per image pixel for the task of background subtraction, Pattern Recognition Letters, Volume 27, No 7, pages 773-780, January 2006.

[147]Cvetkovic S., Bakker P., Schirris J., de With P. Background Estimation and Adaptation Model With Light-Change Removal for Heavily Down-Sampled Video Surveillance Signals", ICIP 2006, pages 1829-1832, 2006

[148]Witherspoon S., Zhang M. Negative Coefficient Polynomial Kerne Density Estimation for Visualization, International Conference on Modeling and Simulation, page 567, Montreal, Canada, June 2007.

[149] Ramezani R., Angelov P. , Zhou X. A Fast Approach to Novelty Detection in Video Streams using Recursive Density Estimation, IEEE Symposium on Intelligent Systems, Volume 2, pages 142-147, Varna, Bulgaria, September 2008.

[150] Mao Y., Shi P. Multimodal background model with noise and shadow suppression for moving object detection, Journal of Southeast University, Volume 20, No.4, pages 423-426, December 2004.

[151] Mao Y., Shi P. Diversity sampling based kernel density estimation for background modeling, Journal of Shanghai University, Volume 9 , Issue 6, Pages 506-509, December 2005.

[152]Tang P., Gao L., Liu Z. Salient Moving Object Detection Using Stochastic Approach Filtering, ICIG 2007, pages 530-535, 2007.

[153] Tavakkoli A., Nicolescu M., Bebis G. An Adaptive Recursive Learning Technique for Robust Foreground Object Detection, ECCV 2006, Graz, Austria, May 2006.

[154]Tavakkoli A., Nicolescu M., Bebis G. Robust Recursive Learning for Foreground Region Detection in Videos with Quasi-Stationary Backgrounds", ICPR 2006, Hong Kong, August 2006.

[155] Sheikh Y., Shah M. Bayesian Modeling of Dynamic Scenes for Object Detection, IEEE Transactions on Pattern Analysis and Machine Intelligence, Volume 27, No. 11, pages 1778-1792, November 2005.

[156] Antic B., Crnojevic V. Joint Domain-Range Modeling of Dynamic Scenes with Adaptive Kernel Bandwidth, ACIVS 2007, 2007.

[157]Mittal A. Motion-Based Background Subtraction using Adaptive Kernel Density Estimation, CVPR 2004, Washington, USA, July 2004.

[158]Parag T., Elgammal A., Mittal A. A Framework for Feature Selection for Background Subtraction, CVPR 2006, June 2006.

[159]Pahalawatta P., Depalov D., Pappas T., Katsaggelos A. Detection, Classification, and Collaborative Tracking of Multiple Targets Using Video Sensors, , IPSN 2003, pages 529-544, Palo Alto, CA, USA April 2003. 
[160]Örten B., Soysal M., Alatan A. Person Identification in Surveillance Video by Combining Mpeg-7 Experts, WIAMIS 2005, pages 352-355, Montreux, Switzerland, April 2005.

[161]Tanaka T., Shimada A., Arita D., Taniguchi R. Object Segmentation under Varying Illumination based on Combinational Background Modeling", Joint Workshop on Machine Perception and Robotics, 2008.

[162] Mahamud S. Comparing Belief Propagation and Graph Cuts for Novelty Detection, CVPR 2006, Volume 1, pages 1154-1159, 2006.

[163]Hu J., Jiang J., Qi M. A Motion Detection Approach Based on Context Modeling, CEPS 2006, pages 78-80, March 2006.

[164]Akman O., Multi-Camera Video Surveillance Detection, Occlusion Handling, Tracking and Event Recognition, PhD Thesis, Middle East Technical University, 2007.

[165]Oliver N., Rosario B., Pentland A. A Bayesian Computer Vision System for Modeling Human Interactions", ICVS 1999, Gran Canaria, Spain, January 1999.

[166] Xu Z., Shi P., Gu I. An Eigenbackground Subtraction Method using Recursive Error Compensation, Advances in Multimedia Information Processing, PCM 2006, Volume 4261, pages 779-787, 2006.

[167]Xu Z., Gu I., Shi P. Recursive error-compensated dynamic eigenbackground learning and adaptive background subtraction in video, Optical Engineering, Volume 47, Issue 5, May 2008.

[168]Kawabata S., Hiura S., Sato K., Real-Time Detection of Anomalous Objects in Dynamic Scene, ICPR 2006, Volume 3, pages $1171-1174$, Hong-Kong, August 2006.

[169]Rymel J., Renno J., Greenhill D. , Orwell J., Jones G. Adaptive EigenBackgrounds for Object Detection, ICIP 2004, Suntec City, Singapore, October 2004

[170]Li Y., Xu L., Morphett J., Jacobs R. An Integrated Algorithm of Incremental and Robust PCA, ICIP 2003, Barcelona, Spain, September 2003.

[171] Li Y. On incremental and robust subspace learning, Pattern Recognition, PR 2004, Volume 37, Issue 7, pages 1509-1518, 2004.

[172] Skocaj D., Leonardis A. Weighted and Robust Incremental Method for Subspace Learning, ICCV 2003, pages 1494-1501, 2003

[173] Skocaj D., Leonardis A., Incremental and robust learning of subspace representations, IVC 2006, pages 1-12, 2006.

[174]Zhang J., Zhuang Y. Adaptive Weight Selection for Incremental Eigen-Background Modeling, ICME 2007, Beijing, China, July 2007.

[175]Wang L., Wang L., Zhuo Q., Xiao H., Wang W. Adaptive Eigenbackground for Dynamic Background Modeling, Intelligent Computing in Signal Processing and Pattern Recognition, Lecture Notes in Control and Information Sciences, Volume 345, pages 670675, 2006.

[176]Wang L., Wang L., Wen M., Zhuo Q., Wang W. Background subtraction using incremental subspace learning, ICIP 2007, Volume 5 , pages 45-48, 2007.

[177]Li R., Chen Y., Zhang X. Fast Robust Eigen-Background Updating For Foreground Detection, ICIP 2006, pages 1833-1836, 2006.

[178]Han B., Jain R. Real-Time Subspace-Based Background Modeling Using Multi-channel Data, ISVC 2007, pages 162-172, November 2007.

[179]Vapnik V. Statistical Learning Theory, Wiley, New York, 1998.

[180]Lin H., Liu T., Chuang J. A probabilistic SVM approach for background scene initialization, ICIP 2002, Volume 3, pages 893-896, Rochester, New York, September 2002.

[181]Smola A., Scholkopf B., A tutorial on support vector regression, NeuroCOLTS Technical Report Series NC2-TR-1998-030, October 1998.

[182] Ma J., Theiler J. Accurate on-line support vector regression, Journal on Neural Computation, Volume 15, pages 2683-2703, 2003

[183]Wang J., Bebis G., Nicolescu M., Nicolescu M., Miller R. Improving target detection by coupling it with tracking, Machine Vision and Application, pages 1-19, 2008.

[184]Wang J, Bebis G., Miller R. Robust Video-Based Surveillance by Integrating Target Detection with Tracking, CVPR 2006, New York, NY, June 2006.

[185]Tax D., Duin R. Support vector data description, Machine Learning, Volume 54, pages 45-66, 2004.

[186]Tavakkoli A., Nicolescu M., Bebis G. A Novelty Detection Approach for Foreground Region Detection in Videos with Quasi-stationary Backgrounds, ISVC 2006, pages 40-49, November 2006.
[187]Tavakkoli A., Ambardekar A., Nicolescu M., Louis S. A Genetic Approach to Training Support Vector Dada Descriptors for Background Modeling in Video Data, ISVC 2007, Lake Tahoe, USA, November 2007.

[188] Tavakkoli A., Nicolescu M., Nicolescu M., Bebis G. Incremental SVDD Training: Improving Efficiency of Background Modeling in Videos, International Conference on Signal and Image Processing, page 623, Kailua-Kona, Hawaii, August 2008.

[189]Tavakkoli A., Nicolescu M., Nicolescu M., Bebis G. Efficien Background Modeling through Incremental Support Vector Data Description, ICPR 2008, Tampa, Florida, December 2008.

[190]Kim H., Sakamoto R., Kitahara I., Toriyama T., Kogure K. Background Subtraction using Generalized Gaussian Family Model”, IET Electronics Letters, Volume 44, Issue 3, pages 189-190, January 2008.

[191]Kim H., Sakamoto R., Kitahara I., Toriyama T., Kogure K. Robust Silhouette Extraction Technique Using Background Subtraction, Meeting on Image Recognition and Understand, MIRU 2007, Hiroshima, Japan, July 2007.

[192]Kim H., Sakamoto R., Kitahara I., Toriyama T., Kogure K. Robust Foreground Extraction Technique Using Gaussian Family Model and Multiple Thresholds, ACCV2007, LNCS 4843, pages 758-768, Tokyo, Japan, November 2007.

[193]Bouguila N., Ziou D. Unsupervised Selection of a Finite Dirichlet Mixture Model: An MML-Based Approach, IEEE Transaction on KDE, Volume 18, pages 993-1009, 2006.

[194]Allili M., Bouguila N., Ziou D. A Robust Video Foreground Segmentation by Using Generalized Gaussian Mixture Modeling, Canadian Conference on Computer and Robot Vision, CRV 2007, pages 503-509, 2007.

[195]Allili M., Bouguila N., Ziou D. Finite Generalized Gaussian Mixture Modelling and Application to Image and Video Foreground Segmentation, Journal of Electronic Imaging, 2008.

[196]Leonardis A., Subspace Methods for Visual Learning and Recognition, Tutorial Session, ECCV 2002, Copenhagen, 2002.

[197]Skočaj D., Leonardis A. Canonical correlation analysis for appearance-based orientation and self-estimation and self-localization, CogVis Meeting, Zurich, Switzerland, January 2004.

[198] Yamazaki M., Xu G., Chen Y. Detection of Moving Objects by Independent Component Analysis, ACCV 2006, pages 467-478, 2006.

[199]Bell A., Sejnowski T., An information-maximization approach to blind separation and blind deconvolution, Neural Computation, Volume 7, Issue 11, pages 1129-1159, 1995.

[200]Tsai D., Lai C. Independent Component Analysis-Based Background Subtraction for Indoor Surveillance, IEEE Transactions on Image Processing, IP 2009, Volume 18, Issue 1, pages 158-167, January 2009.

[201] Schutte J., Groenwold A. A study of global optimization using particle swarms, Jounal of Global Optimization, Volume 31, No. 1, pages 93 $108,2005$.

[202] Bucak S., Gunsel B., Gursoy O. Incremental Non-negative Matrix Factorization for Dynamic background Modelling, International Workshop on Pattern Recognition in Information Systems, Funchal, Portugal, June 2007

[203]Bucak S., Gunsel B. Incremental Subspace Learning and Generating Sparse Representations via Non-negative Matrix Factorization, Pattern Recognition, Volume 42, Issue 5, pages 788-797, 2009.

[204]Li X., Hu W., Zhang Z., Zhang X. Robust Foreground Segmentation Based on Two Effective Background Models, MIR 2008, pages 223228, Vancouver, Canada, October 2008.

[205] Hu W., Li X., Zhang X., Shi X., Maybank S., Zhang Z. Incremental Tensor Subspace Learning and Its Applications to Foreground Segmentation and Tracking, International Journal of Computer Vision, Volume 91, Number 3, pages 303-327, 2011.

[206]Zhao Y., Gong H., Lin L., Jia Y. Spatio-temporal Patches for Night Background Modeling by Subspace Learning, ICPR 2008, 2008.

[207]Wu X., Wang Y., Li J. Video Background Segmentation Using Adaptive Background Models, ICIAP 2009, pages 623-632, September 2009.

[208] Wu X., Yang L., Yang C. Real-time Foreground Segmentation Based on a Fused Background Model, ICCAE 2010, pages 585-588, February 2010. 
[209]Zhang J, Tian Y., Yang Y., Zhu C. Robust Foreground Segmentation Using Subspace Based Background Model, , APCIP 2009, Volume 2, pages 214-217, July 2009.

[210]La X., Zhao G., Meng H. A New Method for Selecting Gradient Weight in Incremental Eigen-Background Modeling, ICIA 2009, pages 801-805, Zhuhai, China, June 2009.

[211] Dong T., Han T., DeSouza G. Illumination invariant foreground detection using multi-subspace learning, Journal International of Knowledge-Based and Intelligent Engineering Systems, Volume 14, Number 1, pages 31-41, 2010.

[212]Dong Y., DeSouza G. Adaptive Learning of Multi-Subspace for Foreground Detection under Illumination Changes, Computer Vision and Image Understanding, 2011.

[213]Kawanishi Y., Mitsugami I., Mukunoki M., Minoh M. Background Image Generation Keeping Lighting Condition of Outdoor Scenes, International Conference on Security Camera Network, Privacy Protection and Community Safety, SPC2009, October 2009.

[214] Kawanishi Y., Mitsugami I., Mukunoki M., Minoh M. Background image generation by preserving lighting condition of outdoor scenes, Procedia - Social and Behavioral Science, Volume 2, No.1, pages 129136, March 2010.

[215] Salas J, Martínez P., Gonzàlez J. Background Updating with the use of Intrinsic Curves, International Conference on Image Analysis and Recognition, ICIAR 2006, Póvoa de Varzim, Portugal, September 2006.

[216]Liang Y., Wang Z., Xu X., Cao X. Background Pixel Classification for Motion Segmentation using Mean Shift Algorithm, ICMLC 2007, pages 1693-1698, Hong Kong, China, 2007.

[217]Zhou D., Zhang H. Modified GMM background modeling and optical flow for detection of moving objects, IEEE International Conference on Systems, Man and Cybernetics, pages 2224-2229, Hawaii, USA, October 2005.

[218]Greiffenhagen M., Ramesh V., Niemann H. The systematic design and analysis cycle of a vision system: A case study in video surveillance, CVPR 2001, 2001.

[219]Campbell-West F., Miller P., Wang H. Independent moving object detection using a color background model, AVSS 2006, Sydney, Australia, November 2006.

[220]Gao X., Boult T., Coetzee F., Ramesh V. Error analysis of background adaption", CVPR 2000, Volume 1, pages 503-510, June 2000.

[221]Xu L. Robust detection and tracking of multiple objects in cluttered scenes, BMVA 2004, March 2004.

[222]Teixeira L., Cardoso J., Corte-Real L. Object segmentation using background modelling and cascaded change detection, Journal of Multimedia, Volume 2, Issue 5, pages 55-65, 2007.

[223] Achkar F., Amer A. Hysteresis-based selective Gaussian mixture models for real-time background maintenance, SPIE Symposium on Electronic Imaging, Conference on Visual Communications and Image, San Jose, CA, USA, January 2007.

[224]Rao N., Di H., Xu G. Joint correspondence and background modeling based on tree dynamic programming", ICPR 2006, 2006, 425-428.

[225]Zen H., Lai S. Adaptive foreground object extraction for real-time video surveillance with lighting variations, ICASSP 2007, Volume 1, pages 1201-1204, 2007.

[226]Utasi A., L. Czúni L. Reducing the Foreground Aperture Problem in Mixture of Gaussians Based Motion Detection, EURASIP Conference Focused on Speech and Image Processing, Multimedia Communications and Services EC-SIPMCS 2007, Maribor, Slovenia, 2007.

[227]Dalley G., Migdal J., Grimson W. Background Subtraction for Temporally Irregular Dynamic Textures, WACV 2008, Colorado, USA, January 2008.

[228]Cuevas C., Salgado L., Garcia N. A new strategy based on adaptive mixture of Gaussians for real-time moving objects segmentation, Real Time image Processing, SPIE 2008, Volume 6811, January 2008.

[229]Cheng S., Luo X., Bhandarkar S. , A Multiscale Parametric Background Model for Stationary Foreground Object Detection, WMVC 2007, Austin, USA, February 2007.

[230]Klare B., Sarka S. Background Subtraction in Varying Illuminations Using an Ensemble Based on an Enlarged Feature Set, OTCBVS 2009, Miami, Florida, June 2009.

[231]Wei S., Jiang S., Huang Q. A Pixel-Wise Local Information-Based Background Subtraction Approach, ICME 2008, pages 1501-1504, April 2008.
[232]Huang J., Chen C. Learning Moving Cast Shadows for Foreground Detection, International Workshop on Visual Surveillance, VS 2008, 2008.

[233]Zhang T., Li S., Xiang S., Zhang L., Liu S. Co-Training Based Segmentation of Merged Moving Objects, International Workshop on Visual Surveillance, VS 2008, 2008.

[234]Tian Y., Feris R., Hampapur A. Real-Time Detection of Abandoned and Removed Objects in Complex Environments" International Workshop on Visual Surveillance, VS 2008, 2008.

[235]Izadi M., Saeedi P. Robust Region-Based Background Subtraction and Shadow Removing Using Colour and Gradient Information, ICPR 2008, pages 1-5, Tampa, USA, 2008.

[236]Rahman J., Motion Detection for Video Surveillance, Master Thesis, Department of Computer Science, Högskolan Dalarna, November 2008

[237] Shimada A., Tanaka T., Arita D., Taniguchi R. Spatial-Temporal Integration of Adaptive Gaussian Mixture Background Models, KoreaJapan Joint Workshop on Frontiers of Computer Vision, FCV 2008, 2008.

[238]Niu L., Jiang N. A Moving Objects Detection Algorithm Based on Improved Background Subtraction, ISDA 2008, Volume 03, pages 604-607, 2008.

[239]Wang W., Yang J., Gao W. Modeling Background and Segmenting Moving Objects from Compressed Video, IEEE Transactions on Circuits and Systems for Video Technology, Volume18, Issue 5, pages 670-681, May 2008

[240] Shahid H., Khan K., Qazi W. Using modified mixture of Gaussians for background modeling in video surveillance, ICAST 2008, Islamabad, Pakistan, November 2008.

[241]Ju S., Chen X., Xu G. An Improved Mixture Gaussian Models to Detect Moving Object under Real-time Complex Background, ICC 2008, pages 730-734, 2008.

[242]Zhong B., Yao H., Shan S., Chen X., Gao W. Hierarchical Background Subtraction using Local Pixel Clustering, ICPR 2008, Florida, USA, December 2008.

[243]Cai X., Jiang L., Hao X., Meng X. A new region Gaussian background model for video surveillance, International Conference on Natural Computation, pages 123-127, 2008.

[244]Yuan B. , Sun Z. Guide background model update with object tracking, Journal of Computational Information Systems, Volume 4, Issue 4, pages 1635-1642, August 2008.

[245] Singh A., Jaikumar P., Mitra S., Joshi M., Banerjee A. Detection and Tracking of Objects in Low Contrast Conditions, National Conference on Computer Vision, Pattern Recognition, Image Processing and Graphics, NCVPRIPG 2008, pages 98-103, India, January 2008.

[246]Singh A., Jaikumar P., Mitra S., Joshi M., Banerjee A. Background Subtraction in Videos using Adaptive Mixture Models with Split-andMerge Operation, Journal of the National Academy of Sciences, India

[247]Jaikumar P., Singh A., Mitra S. Background Subtraction in Videos using Bayesian Learning with Motion Information, BMVC 2008, pages 615-624, Leeds, UK, September 2008

[248] Singh A., Mitra S. Background Subtraction in Videos using Bayesian Learning of Gaussian Mixture Models, IEEE Transactions on Image Processing, 2009.

[249]Shimada A., Taniguchi R. Object Detection Based on Gaussian Mixture Predictive Background Model under Varying Illumination, International Workshop on Computer Vision, MIRU 2008, July 2008

[250]Shimada A., Taniguchi R. Object Detection Based on Fast and LowMemory Hybrid Background Model, IEEJ Transactions on Electronics, Information and Systems, Volume 129-C, Number 5, pages 846-852, May 2009.

[251]Lien C., Hua C., Jiang Y., Jang L. Large Area Video Surveillance System with Handoff Scheme among Multiple Cameras, MVA 2009, Yokohama, Japan, May 2009.

[252]Wang F., Dai S. Adaptive Background Update Based on Mixture Models of Gaussian, ICIA 2009, Zhuhai, China, June 2009.

[253] Li Y., Xiong C., Yin Y., Liu L. Moving Object Detection Based on Edged Mixture Gaussian Models", ISA 2009, pages 1-5, May 2009.

[254]Huang T., Qiu J., Sakayori T., Goto S., T. Ikenaga T. Motion Detection Based on Background Modeling and Performance Analysis for Outdoor Surveillance", International Conference on Computer Modeling and Simulation, ICCMS 2009, pages 38-42, Macau, February 2009. 
[255]Huang T., Qiu J., Sakayori T., Ikenaga T. Robust Background Segmentation using Background Models for Surveillance Application, MVA 2009, pages 402-405, 2009.

[256]Cai X., Ali F., Stipidis E. Background Modeling for Detecting Movethen-stop Arbitrary-long Time Video Objects, WIAMIS 2009, London, UK, May 2009.

[257]Chang L., Hsu W. Foreground segmentation for static video via multicore and multi-modal graph cut, ICME 2009, New York, USA, July 2009.

[258]Al Najjar M., Ghosh S., Bayoumi M. A Hybrid Adaptive Scheme Based on Selective Gaussian Modeling for Real-Time Object Detection, ISCAS 2009, pages 936-939, Taipei, Taiwan, May 2009.

[259]Hu H., Li Z., Qu Z., Wang D. Vision-Based Moving Objects Detection with Background Modeling, International Conference on Measuring Technology and Mechatronics Automation, ICMTMA 2009, Volume 2, pages 436-439, 2009

[260]Chen Z., Pears N., Freeman M., Austin J. Background subtraction in video using recursive mixture models, spatio-temporal filtering and shadow removal, ISVC 2009, Las Vegas, December 2009.

[261] Xuehua S., Yu C., Jianfeng G., Jingzhu C. A Robust Moving Objects Detection Algorithm Based on Gaussian Mixture Model, ITSC 2009, Volume 1, pages 566-569, 2009.

[262]Rui Y., Xuehua S., Shu Y., Moving object detection based on an improved Gaussian mixture background model, ISECS International Colloquium on Computing, Communication, Control, and Management, CCCM 2009, Volume 1, pages 12-15, August 2009.

[263]Landabaso J., Pujol J., Montserrat T., Marimon D., Civit J. , Escoda O. A Global Probabilistic Framework for the Foreground, Background and Shadow Classification Task”, ICIP 2009, pages 3189-3192, 2009.

[264]Zhong B., Liu S., Yao H., Zhang B., Multi-Resolution Background Subtraction for Dynamic Scenes, ICIP 2009, pages 3193-3196, Cairo, Egypt, November 2009.

[265]Li H., Achim A., Bull D. GMM-based efficient foreground detection with adaptive region update, ICIP 2009, pages 3181-3184, November 2009.

[266]Park D., Byun H. Object-Wise Multilayer Background Ordering for Public Area Surveillance, AVSS 2009, September 2009.

[267]Zheng, H. Liu Z., Wang X. Video Segmentation Method with Integrated Multi-features Based on GMM, International Conference on Intelligence for Modelling Control and Automation, pages 260-264, December 2008.

[268]Zheng H., Liu Z., Wang X. , Video Segmentation Method with Integrated Multi-features Based on GMM, International Conference on Digital Image Processing, DIP 2009, pages 62-66, March 2009.

[269]Li J., Shao C., Xu W., Dong C. Real-Time Pedestrian Detection Based on Improved Gaussian Mixture Model, ICMTMA 2009, Volume 3, pages 269-272, April 2009.

[270]Guo H., Dou Y., Tian T., Zhou J., Yu S. A Robust Foreground Segmentation Method by Temporal Averaging Multiple Video Frames, ICALIP 2008, pages 878-882, 2008.

[271]Krishna R., McCusker M., O'Connor N. Optimising resource allocation for background modeling using algorithm switching, International Conference on Distributed Smart Cameras, ICDSC 2008 pages 1-7, Palo Alto, CA, USA, September 2008.

[272] Sheng Z., Cui X. An adaptive learning rate GMM for background extraction, Optoelectronics Letters, Volume 4, Number 6, pages 460463, November 2008.

[273]Lee D., Ahn J., Kim C. Fast Background Subtraction Algorithm using Two-Level Sampling and Silhouette Detection, ICIP 2009,pages 3177 3180, Cairo, Egypt, November 2009.

[274]Li Q., Zhang Y. Improvement on adaptive mixture Gaussian background model, Computer Application, Volume 7, pages 20142017, 2007.

[275] Wang Z., Tang X. Adaptive background mixture model and shadow removal for traffic images, Joint Conference on Information Sciences, CVPRIP-IV, pages 916-922, Salt Lake City, USA, July 2007

[276]Wang W., Gao W., Wang R. A local hierarchical approach for background modeling and moving targets detection, MIPPR 2009: Automatic Target Recognition and Image Analysis, Proceedings of the SPIE, Volume 7495, pages 1-6, 2009.

[277]Liu J., Zhang D. The Updating Algorithm of Adaptive Gaussian Mixture Background Model, 2006.

[278]Wang J., He F., Zhang X., Gao Y. A Moving Objects Detection Algorithm using Iterative Division and Gaussian Mixture Model",
International Conference on Advanced Computer and Control, ICACC 2010, Volume 5, pages 229-233, 2010.

[279] Wang Y., Liang Y., Pan Q., Cheng Y., Zhao C. Spatiotemporal Background Modeling Based On Adaptive Mixture Of Gaussians, Acta Automatica Sinica,Volume 35, No. 4, April 2009.

[280]Liu X., Liu H., Qiang Z., Geng X. Adaptive Background Modeling Based on Mixture Gaussian Model and Frame Subtraction, Journal of Image and Graphics, April 2008

[281]Yuan C., Wang C., Zhang X., Liu Y. Video Segmentation of Illuminance Abrupt Variation Based on MOGs and Gradient Information, Journal of Image and Graphics, November 2007.

[282]Zhong B., Yao H., Liu S. Robust Background Modeling via Standard Variance Feature", ICASSP 2010, March 2010.

[283] Baloch T. Background Subtraction in Highly Illuminated Indoor Environment, Master Thesis, Department of Computer Science and Engineering, Indian Institute of Technology, Kanpur, India, 2010.

[284]Quast K., Obermann M., Kaup A. Real-time Moving Object Detection in Video Sequences using spatio-temporal adaptive Gaussian Mixture Models", VISAPP 2010, pages 413-418, Angers, France, May 2010.

[285] Molin J. Foreground Segmentation of Moving Objects, Master Thesis, Department of Electrical Engineering, Linköpings University, Sweden, 2010.

[286] Qin B., Wang J., Gao J., Pang T., Su F. A Traffic Video Background Extraction Algorithm Based on Image Content Sensitivity, ICSI 2010, pages 603-610, 2010.

[287]Yan Q., Xu Y., Yang X., Traversoni L. Real-Time Foreground Detection Based on Tempo-Spatial Consistency Validation and Gaussian Mixture Model, IEEE International Symposium on Broadband Multimedia Systems and Broadcasting, BMSB 2010, pages 1-4, Shangai, China, March 2010.

[288]Huang T., Fang X., Qiu J., Ikenaga T. Adaptively Adjusted Gaussian Mixture Models for Surveillance Applications, MMM 2010, LNCS 5916, pages 689-694, 2010

[289] Mohamed S., Tahir N., Adnan R. Background Modelling and Background Subtraction Performance for Object Detection, CSPA 2010,2010

[290] Yu J., Zhou X., Qian F., Object Kinematic Model: A Novel Approach of Adaptive Background Mixture Models for Video Segmentation, WCICA 2010, pages 6225-6228, Jinan, China, July 2010.

[291]Li Z., Zhong L., Liu Y., Efficient Foreground Layer Extraction in Video, PCM 2010, Part I, LNCS 6297, pages 319-329, 2010.

[292]Huang C., Wu R., A Multi-layer Scene Model for Video Surveillance Applications, Pacific Rim Conference on Multimedia, PCM 2010, Part I, LNCS 6297, pages 68-79, 2010.

[293]Forczmanski P., Seweryn M. Surveillance Video Stream Analysis Using Adaptive Background Model and Object Recognition, ICCVG 2010, Part I, LNCS 6374, pages 114-121, 2010.

[294]Zhang Y., Bai Y., Zhao S. Moving Object Detection Based on Gaussian Mixture Model within the Quotient Space Hierarchical Theory, Rough Set and Knowledge Technology, RSKT 2010, LNAI 6401, pages 772-777, 2010

[295]Park J, Lee G., Toan N., Cho W., Park S. Moving Object Detection Using Clausius Entropy and Apdative Gaussian Mixture Model, Journal of The Institute of Electronics Engineers of Korea, Volume 47 No. 1, January 2010

[296]Park J, Lee G., Toan N., Cho W., Park S. Moving Object Detection based on Clausius Entropy, CIT 2010, pages 517-521, June 2010.

[297]Suhr J., Jung H., Li G., Kim J. Mixture of Gaussians-based Background Subtraction for Bayer-Pattern Image Sequences, IEEE Transactions on Circuits and Systems for Video Technology, CSVT 2010, 2010.

[298] Shah M., Deng J., Woodford B. Localized Adaptive Learning of Mixture of Gaussians Models for Background Extraction, ICVNZ 2010, Queenstown, New Zealand, November 2010.

[299]Fabián T. Mixture of Gaussians Exploiting Histograms of Oriented Gradients for Background Subtraction, ISVC 2010, Las Vegas, USA, November 2010.

[300] Hu L, Liu W., Li B., Xing W. Robust motion detection using histogram of oriented gradients for illumination variations, ICIMA 2010, Volume 2, pages 443-447, Wuhan, China, May 2010.

[301]He S., Guan Q., Xu S., Li Y., Wu Y. Improving mixture Gaussian background model by integrating trace information obtained from Kalman filter, ICCCAS 2010, pages 378-382, Chengdu, China, July 2010. 
[302]Kan J., Tang J., Li K., Ou X. Background modeling method based on improved multi-Gaussian distribution, International Conference on Computer Application and System Modeling, ICCASM 2010, Volume 2, pages 214-218, Taiyuan, China, October 2010.

[303]Qu Z., Yu M., Liu J. Real-time traffic vehicle tracking based on improved MoG background extraction and motion segmentation, ISSCAA 2010, pages 676-680, Harbin, China, June 2010.

[304]Fazli S., Pour H., Bouzari H. A Novel GMM-Based Motion Segmentation Method for Complex Background, GCC 2009, Kuwait, March 2009.

[305]Fazli S., Pour H., Bouzari H. Multiple Object Tracking Using Improved GMM-Based Motion Segmentation, ECTI-CON 2009, Pataya, Thailand, May 2009.

[306]Fazli S., Pour H., Bouzari H. A robust hybrid movement detection method in dynamic background, ECTI-CON 2009, pages 1134-1137, Pataya, Thailand, May 2009.

[307]Wang W., Qian H., Chen P., Chen S. High level feedback for foreground detection, YC-ICT 2009, pages 323-326, Beijing, China, September 2009.

[308]Quast K., Kaup A. AUTO GMM-SAMT: An Automatic Object Tracking System for Video Surveillance in Traffic Scenarios, EURASIP Journal on Image and Video Processing, Volume 2011, Article ID 814285, 14 pages, 2011.

[309]Lin H., Chuang J., Liu T., Regularized Background Adaptation: A Novel Learning Rate Control Scheme for Gaussian Mixture Modeling, IEEE Transaction on Image Processing, 2011.

[310]Qi Y., Wang Y. Memory-based Gaussian Mixture Modeling for Moving Object Detection in Indoor Scene with Sudden Partial Changes, ICSP 2010, pages 752-755, 2010.

[311]Qi Y., Wang Y., Li Y. Memory-based Gaussian Mixture Background Modeling, Acta Automatica Sinica, Volume 36, No. 11, pages 15201526, November 2010.

[312]Xue G., Sun J, Song L. Background Subtraction based on Phase and Distance Transform under Sudden Illumination Change, ICIP 2010, pages 3465-3468, Hong Kong, China, September 2010.

[313]Feldmann T., Diesselberg L., Worner A. Adaptive Foreground/Background Segmentation Using Multiview Silhouette Fusion”, DAGM 2009, LNCS 5748, pages 522-531, 2009.

[314]Feldmann T. Spatio-Temporal Optimization for Foreground/Background Segmentation, International Workshop on Visual Surveillance, VS 2010, Queenstown, New Zealand, November 2010.

[315]Li D., Dawei L., Goodman E. Online background learning for illumination-robust foreground detection, ICARCV 2010, page 1093, Singapore, Singapore, December 2010.

[316]Dickinson P., Hunter A., Appiah K. Segmenting video foreground using a multi-class MRF, ICPR 2010, pages 1848-1851, Istanbul, Turkey, August 2010.

[317]Zhou H., Zhang X., Gao Y., Yu P. Video background subtraction using improved Adaptive-K Gaussian Mixture Model, ICACTE 2010, Chengdu, China, August 2010.

[318]Tian Y., Wang X. A fast convergent Gaussian mixture model in moving object detection with shadow elimination, ICEEE 2010, Henan, China, November 2010

[319]Li L., Xu J. Moving human detection algorithm based on Gaussian mixture model, Chinese Control Conference, CCC 2010, pages 28532856, 2010

[320]Bin Z., Liu Y. Robust Moving Object Detection and Shadow Removing Based on Improved Gaussian Model and Gradient Information, ICMT 2010, Ningbo, China, October 2010.

[321]Liu Y., Bin Z. The improved moving object detection and shadow removing algorithms for video surveillance", CISE 2010, Wuhan, China, December 2010

[322]Zhao L., He X. Adaptive Gaussian mixture learning for moving object detection, Conference on Broadband Network and Multimedia Technology, IC-BNMT2010, pages 1176-1180, Beijing, China, October 2010

[323]Li Y., Tian H., Zhang Y. An improved Gaussian mixture background model with real-time adjustment of learning rate, ICINA 2010 , Volume1, pages 1512-1515, 2010.

[324]Lai C., Chen S., J. Wang J. Gaussian Mixture of Background and Shadow Model, IPPR Conference on Vision, Graphics and Image Processing, CVGIP 2010, August 2010.
[325] Yang J., Wang J., Lu H. A Hierachical Approach for Background Modeling and Moving Objects Detection, International Journal of Control, Automation and Systems, pages 940-947, 2010.

[326]Shao J., Jia Z., Li Z., Liu F., Zhao J., Peng P. A Closed-loop Background Subtraction Approach for Multiple Models based Multiple Objects Tracking” Journal of Multimedia, Volume 6, No. 1, pages 33-38, February 2011.

[327]Zhang X., Zhou J. Moving target detection in complex scenes based on spatio-temporal domain analysis, CISP 2010, pages 1520-1523, Yantai, China, October 2010.

[328] Wang Z., Xu H., Sun L., Yang S. Background Subtraction in Dynamic Scenes with Adaptive Spatial Fusing", IEEE International Workshop on Multimedia Signal Processing, pages 1-6, Rio de Janeiro, Brazil, October 2009.

[329] Wang S., Su T., Lai S. Detecting moving objects from dynamic background with shadow removal, ICASSP 2011, Prague, Czeh Republic, May 2011.

[330]Zou W., Zhao D., Sun G., Yu Z., Wang Y. An improved method of target detection based on Gaussian Mixture Model and Average Background Method, SPPRA 2011, Innsbruck, Austria, February 2011.

[331]Wang H., Miller P. Regularized Online Mixture of Gaussians for background subtraction, AVSS 2011, Klagenfurt, Austria, September 2011.

[332] Culbrik D., Marques O., Socek D., Kalva H., Furht B. Neural network approach to background modeling for video object segmentation", IEEE Transaction on Neural Networks, Volume 18, No. 6, pages 1614-1627, 2007

[333] Maddalena L., Petrosino A. A self organizing approach to background subtraction for visual surveillance applications, IEEE Transactions on Image Processing, Volume17, No. 7, pages 1729-1736, 2008

[334]Bouwmans T. Subspace Learning for Background Modeling: A Survey, Recent Patents on Computer Science, Volume 2, No 3, pages 223-234, November 2009.

[335] Sivabalakrishnan M., Manjula D., Adaptive Background subtraction in Dynamic Environments Using Fuzzy Logic, International Journal on Computer Science and Engineering, Volume 02, No. 2, pages 270273, 2010.

[336]Biswas S., Sil J., Sengupta N. Background Modeling and Implementation using Discrete Wavelet Transform: a Review, JICGST-GVIP, Volume 11, Issue 1, pages 29-42, March 2011.

[337]Liu Z., Chen W., Huang K., Tan T. A Probabilistic Framework Based on KDE-GMM Hybrid Model for Moving Object Segmentation in Dynamic Scenes, VS 2008, 2008.

[338]Ding J., Li M., Huang K., Tan T. Modeling Complex Scenes for Accurate Moving Objects Segmentation, ACCV 2010, pages 82-94, 2010.

[339]Hao J., Li C., Xiong Z., Hussain E. A temporal-spatial background modeling of dynamic scenes, Frontiers Computational Science China, 2011.

[340]Qiu C., Vaswani N. Real-time Robust Principal Components Pursuit' International Conference on Communication Control and Computing, 2010

[341]Qiu C., Vaswani N. Support Predicted Modified-CS for Recursive Robust Principal Components' Pursuit, IEEE International Symposium on Information Theory, ISIT 2011, 2011

[342]Qiu C., Vaswani N. ReProCS: A Missing Link between Recursive Robust PCA and Recursive Sparse Recovery in Large but Correlated Noise, Preprint, 2011.

[343]Candes E., Li X., Ma Y., Wright J., Robust Principal Component Analysis?, 2009.

[344]Wright J., Peng Y., Ma Y., Ganesh A., Rao S. Robust Principal Component Analysis: Exact Recovery of Corrupted Low-Rank Matrices by Convex Optimization, Neural Information Processing Systems, NIPS 2009, December 2009

[345] Gu J., Liu Z., Zhang Z. Novel moving object segmentation algorithm using kernel density estimation and edge information, Journal of Computer-Aided Design and Computer Graphics, Volume 21, Issue 2, pages 223-228, February 2009.

[346] Martel-Brisson N., Zaccarin A. Unsupervised Approach for Building Non-Parametric Background and Foreground Models of Scenes with Significant Foreground Activity", ACM Workshop on Vision Networks for Behavior Analysis, VNBA 2008, pages 93-100, Vancouver, British Columbia, Canada, October 2008. 
[347]Sung K., Hwang Y., Kweon I. Robust Background Maintenance for Dynamic Scenes with Global Intensity Level Changes, International Conference on Ubiquitous Robots and Ambient Intelligence, URAI 2008, pages 759-762, 2008

[348]Hwang Y., Sung K., Chae J., Park Y., Kweon I. Robust background maintenance by estimating global intensity level changes for dynamic scenes, Intelligent Service Robotics, ISR 2009, Volume 2, No. 3, pages 187-194, 2009.

[349]Elgammal A. Efficient Non parametric Kernel Density Estimation for Real-time Computer Vision, PhD Thesis, Department of computer science, University of Maryland, College Park, 2002.

[350]Sadeghi P., Angelov P., Ramezani R. A fast approach to autonomous real-time novelty detection, multi objects identification and tracking in video stream using recursive density estimation, IPMU 2010, pages 30-43, Dortmund, Germany, July 2010.

[351]Quivy C., Kumazawa I. Background Images Generation Based on the Nelder-Mead Simplex Algorithm Using the Eigenbackground Model, ICIAR 2011, pages 21-29, June 2011.

[352]Zhang Z., Lipton A., Venetianer P., Yin W. Background modeling with feature blocks, US 075136, 2008.

[353]Brown L., Hampapur A., Lu Z., Senior A., Tian Y., "Robust and efficient foreground analysis for real-time video surveillance", US 7620266, 2009.

[354]Mittal A., Ramesh V., Monnet A. Method for scene modeling and change detection, US 7574019, 2009.

[355]Meng L., Wu W., Li Y. System and method for segmenting foreground and background in a video", US 0098331, 2010.

[356]Chang F., Hsu W., Chueh M. Method and system for foreground detection using multi-modality fusion graph cut, US 0208987, 2010.

[357]Chang F., Hsu W., Chueh Method and system for foreground detection using multi-modality fusion graph cut, GB 2467804, 2010.

[358]Xie D. Background model initializing and updating method based on video monitoring, CN 101489121, 2009.

[359]Han B., Comaniciu D., Zhu Y., Zhou X. System and method for sequential kernel density approximation through mode propagation, US 7480079, 2009.

[360]Zhu S., Zhao Y. Background modeling method based on space-time video block and online subspace learning, CN 101645171, 2010

[361]Butler D., Sridharan S. Real-Time Adaptive Background Segmentation, ICASSP 2003, 2003.

[362]Kim K., Chalidabhongse T., Harwood D., Davis L. Real-time Foreground-Background Segmentation using Codebook Model, RealTime Imaging, 2005. 